\title{
THE SUBOPTIMAL NEHARI PROBLEM FOR WELL-POSED LINEAR SYSTEMS*
}

\author{
RUTH F. CURTAIN ${ }^{\dagger}$ AND MARK R. OPMEER ${ }^{\dagger}$
}

\begin{abstract}
We solve the suboptimal Nehari problem for a transfer function that has a state-space realization as a system-stable (input, output and input-output stable) well-posed linear system. We obtain an explicit solution in terms of the state-space parameters.
\end{abstract}

Key words. infinite-dimensional linear systems, J-spectral factorizations, well-posed linear systems, stability, Hankel operators, Lyapunov equations, Nehari problem

AMS subject classifications. 41A30, 47B35, 47N70, 93B28

DOI. $10.1137 /$ S036301290444318X

1. Introduction. The solution to the optimal Nehari problem is well known. The vector-valued case was solved by Page [22] (see also Nikol'skii [20] and Peller [23]):

$$
\inf _{\mathbf{K}(-s) \in \mathbf{H}_{\infty}(\mathcal{L}(U, Y))}\|\mathbf{G}+\mathbf{K}\|_{\infty}=\left\|H_{\mathbf{G}}\right\|
$$

where $\mathbf{G} \in \mathbf{L}_{\infty}(\mathcal{L}(U, Y)), U, Y$ are separable Hilbert spaces, and $H_{\mathbf{G}}$ is the Hankel operator associated with the symbol $\mathbf{G}$. The suboptimal problem is to find for any $\sigma>\left\|H_{\mathbf{G}}\right\|$ all solutions $\mathbf{K}(-s) \in \mathbf{H}_{\infty}(\mathcal{L}(U, Y))$ to

$$
\|\mathbf{G}+\mathbf{K}\|_{\infty} \leq \sigma .
$$

The suboptimal Nehari problem for functions on the disc has been solved in Kheifets [18] (see also Peller [23]), but for control applications we require explicit solutions in terms of state-space parameters of the continuous-time system as we explain below.

A crucial step in many control problems is solving the suboptimal Nehari problem for the stable case: $\mathbf{G} \in \mathbf{H}_{\infty}(\mathcal{L}(U, Y))$. In Salamon [26] it was shown that any $\mathbf{G} \in \mathbf{H}_{\infty}(\mathcal{L}(U, Y))$ has a system-stable well-posed realization; i.e., there exists a state space $Z$ and (in general unbounded) operators $A, B, C$, where $A$ is the infinitesimal generator of a strongly continuous semigroup on the separable Hilbert space $Z$ and the following stability assumptions are satisfied:

$$
C(s I-A)^{-1} z \in \mathbf{H}_{2}(Y), \quad B^{*}\left(s I-A^{*}\right)^{-1} z \in \mathbf{H}_{2}(U) \quad \forall z \in Z .
$$

$\mathbf{G}$ is the transfer function of the well-posed linear system in the sense that

$$
\mathbf{G}(s)-\mathbf{G}(\alpha)=(\alpha-s) C(s I-A)^{-1}(\alpha I-A)^{-1} B
$$

for all $\alpha$ and $s$ in some open right half-plane. Conversely, every system-stable wellposed linear system has a transfer function in $\mathbf{H}_{\infty}(\mathcal{L}(U, Y))$. Usually in control applications not $\mathbf{G}$, but $A, B$, and $C$ are given, and one wants a solution $\mathbf{K}$ in terms of these state-space parameters.

\footnotetext{
* Received by the editors April 22, 2004; accepted for publication (in revised form) January 18, 2005; published electronically September 20, 2005.

http://www.siam.org/journals/sicon/44-3/44318.html

${ }^{\dagger}$ Mathematics Institute, University of Groningen, P.O. Box 800, 9700 AV Groningen, The Netherlands (curtain@math.rug.nl, opmeer@math.rug.nl).
} 
Recently, it has been shown in Curtain and Sasane [9] that if $\rho(A) \cap i \mathbb{R} \neq \emptyset$, the Nehari problem for a system-stable well-posed linear system can be reduced to solving the Nehari problem for its reciprocal system which has bounded $B$ and $C$ operators. (The reciprocal approach to well-posed linear systems was introduced in Curtain [10] and [11].) Since problems with bounded $B$ and $C$ operators are technically simpler, we first consider the special case of bounded input and output operators. The next step is to use this special case to solve the general case.

Our approach to solving the suboptimal Nehari problem is to obtain solutions $\mathbf{K}$ via the $\mathrm{J}$-spectral factorization problem of finding $\mathbf{X}$ such that

$$
\mathbf{P}^{*}(i \omega) J_{\sigma} \mathbf{P}(i \omega)=\mathbf{X}(i \omega) J_{1} \mathbf{X}(i \omega)^{*} \text { for almost all } \omega \in \mathbb{R},
$$

where

$$
\mathbf{P}(s):=\left[\begin{array}{cc}
I_{Y} & \mathbf{G}(s) \\
0 & I_{U}
\end{array}\right] \text { and } J_{\sigma}:=\left[\begin{array}{cc}
I_{Y} & 0 \\
0 & -\sigma^{2} I_{U}
\end{array}\right]
$$

The candidate solution for $\mathbf{X}$ involves the solutions of the Lyapunov equations

$$
A L_{1}+L_{1} A^{*}=-B B^{*}, \quad A^{*} L_{2}+L_{2} A=-C^{*} C .
$$

The smallest bounded nonnegative solutions are $L_{B}, L_{C}$, the controllability and observability gramians of the system $\Sigma(A, B, C, 0)$, respectively. These are not necessarily the only bounded nonnegative solutions. Once it is shown that $\mathbf{X}$ is indeed a solution to (1.2), the rest of the proof is relatively straightforward and one obtains a solution in terms of the known system parameters $A, B, C, L_{1}, L_{2}$, and $\sigma$.

There have been several versions of this approach in the literature; all but one (Curtain and Oostveen [6]) assume that $A$ is the generator of an exponentially stable $C_{0}$-semigroup. We mention Curtain and Zwart [4], Glover, Curtain, and Partington [14], Ran [24], Curtain and Ran [2], Foias and Tannenbaum [13], Curtain and Zwart [3], and Curtain and Ichikawa [5], who all treat the problem under the assumption that $A$ is the generator of an exponentially stable $C_{0}$-semigroup and varying additional assumptions.

For exponentially stable systems one can, since $i \mathbb{R} \subset \rho(A)$, verify directly that (1.2) holds for all $\omega \in \mathbb{R}$. However, there exist many systems with a stable transfer function for which $A$ does not generate an exponentially stable $C_{0}$-semigroup. This motivated Curtain and Oostveen [6] to consider the class of system-stable systems satisfying (1.1) with bounded $B$ and $C$ and finite-dimensional $U$ and $Y$. Now assumptions (1.1) provide no information about the spectrum of $A$ and so it is not possible to verify (1.2) by a direct calculation. Unfortunately, this point was overlooked in [6]. We give an example of a system-stable system for which the candidate solution $\mathbf{X}$ does not satisfy (1.2) for a certain pair of solutions $L_{1}, L_{2}$ to the Lyapunov equations (1.3). This does not show that the claim in [6] is incorrect, since the claim in [6] is made only for the smallest solutions $L_{B}$ and $L_{C}$. However, this counterexample does show that there is a gap in the proof in [6]. An easy remedy is to make an additional assumption on the spectrum of $A$, e.g., assume that $\sigma(A) \cap i \mathbb{R}$ has measure zero or that $\mathbb{C}_{0}^{+} \subset \rho(A)$. Our major contribution is to show that if $U$ and $Y$ are finite-dimensional, then these assumptions are unnecessary. This new result has consequences for the recent paper by Ball, Mikkola, and Sasane [1] on the NehariTakagi problem, which is a generalization of the suboptimal Nehari problem. Using a J-spectral factorization approach, they solve the suboptimal Nehari-Takagi problem 
for finite-dimensional $U$ and $Y$ under our assumptions (1.1) plus an assumption on the spectrum of $A$. Our result shows that the latter assumption is redundant.

Summarizing, under the assumptions (1.1), for any $\sigma>r^{1 / 2}\left(L_{1} L_{2}\right)$ (here $r(T)$ is the spectral radius of the operator $T$ ) we give an explicit formula for a spectral factor X satisfying (1.2) in terms of the system parameters $A, B, C, L_{1}$, and $L_{2}$ under either of the following additional assumptions:

A1. $\sigma(A) \cap i \mathbb{R}$ has measure zero.

A2. $U$ and $Y$ are finite-dimensional, and $L_{1}$ and $L_{2}$ are chosen to be the controllability and observability gramians $L_{B}$ and $L_{C}$, respectively.

This leads to our second main result: a solution of the suboptimal Nehari problem in terms of the system parameters $A, B, C, L_{1}, L_{2}$, and $\sigma$ under either of the above assumptions A1 or A2.

Our last main result is the extension of this result to the class of system-stable well-posed linear systems satisfying the assumption $\rho(A) \cap i \mathbb{R} \neq \emptyset$ and either assumption A1 or A2. We remark that in the well-posed case the standard formulas for the solution need not be well-defined, but we obtain alternative explicit formulas in terms of the reciprocal system as in Curtain and Sasane [9].

The paper is written to be as self-contained as possible. In section 2 , we summarize relevant known results on state linear systems and in section 3 we prove some interesting new ones. Section 4 contains results on Riccati equations in terms of the concepts of input and output stability and stabilizability. In addition, we study two interesting Riccati equations connected to the Nehari problem. In section 5 we give an example of a system-stable system for which the candidate solution $\mathbf{X}$ does not satisfy (1.2) for a certain pair of solutions $L_{1}, L_{2}$ of the Lyapunov equations. However, we show that in the case that $L_{1}=L_{B}$ and $L_{2}=L_{C}$, we can always construct a spectral factor of (1.2). We collect several of its properties that enable us to obtain a solution of the suboptimal Nehari problem in section 6 . In section 7 , we obtain a parametrization of a family of solutions to the suboptimal Nehari problem for a system-stable state linear system. Finally, in section 8 we recall the concepts of system-stable wellposed linear systems and their reciprocals from [11]. Using the reciprocal approach from [9] we extend our results to obtain an explicit solution of the suboptimal Nehari problem for the class of system-stable well-posed linear systems under the assumption that $\rho(A) \cap i \mathbb{R} \neq \emptyset$ and either of the assumptions $\mathrm{A} 1$ or $\mathrm{A} 2$.

An interesting open question is whether our conclusions also hold if in assumption A2 we allow $U$ and $Y$ to be infinite-dimensional. The existence of frequency domain solutions is also known for this case (see Kheifets [18] or Peller [23]).

2. State linear systems: Known results. First we recall several known results for systems with bounded input and output operators. $A$ is the generator of a strongly continuous semigroup $T(\cdot)$ on a separable Hilbert space $Z, B \in \mathcal{L}(U, Z), C \in$ $\mathcal{L}(Z, Y), D \in \mathcal{L}(U, Y)$ with $U, Y$ separable Hilbert spaces. Following the terminology in Curtain and Zwart [4] we call $\Sigma(A, B, C, D)$ a state linear system. We now define the transfer function and the characteristic function of a state linear system.

Definition 2.1. The transfer function $\mathbf{G}$ is defined as follows: $\mathbf{G}-D$ equals the Laplace transform of $C T(t) B$ on some right half-plane. We define the characteristic function $\mathfrak{G}$ for all $s \in \rho(A)$ by $\mathfrak{G}=D+C(s I-A)^{-1} B$.

Remark 2.2. For $s$ in some right half-plane we have $\mathbf{G}=\mathfrak{G}$, but they may differ outside this region. For a counterexample see Curtain and Zwart [4, Example 4.3.8]. A more detailed discussion is given in Zwart [33].

We introduce a stability concept that is weaker than exponential stability but 
stronger than input-output stability. We will show that this is the right stability concept for the Nehari problem.

Definition 2.3. The state linear system $\Sigma(A, B, C, D)$ is system-stable if

- it is input stable: there exists a constant $\beta>0$ such that for all $u \in$ $\mathbf{L}_{2}(0, \infty ; U),\left\|\int_{0}^{\infty} T(t) B u(t) \mathrm{d} t\right\|^{2} \leq \beta \int_{0}^{\infty}\|u(t)\|^{2} \mathrm{~d} t ;$

- it is output stable: there exists a constant $\gamma>0$ such that for all $z \in Z$, $\int_{0}^{\infty}\|C T(t) z\|^{2} \mathrm{~d} t \leq \gamma\|z\|^{2}$

- it is input-output stable: the transfer function $\mathbf{G} \in \mathbf{H}_{\infty}(\mathcal{L}(U, Y))$.

Remark 2.4. We note that other authors may require the additional assumption that the semigroup be uniformly bounded for $t>0$ for system stability (see Staffans [30] or Mikkola [19, Definition 6.1.1]). The essential difference in our definition is that we have made no stability assumptions on $A$ and so it can have spectrum in the right half-plane $\mathbb{C}_{0}^{+}$.

DeFINITION 2.5. The output map $\mathcal{C}: Z \rightarrow \mathbf{L}_{2}(0, \infty ; Y)$ of an output stable state linear system $\Sigma(A, B, C, D)$ is defined by $(\mathcal{C} z)(t):=C T(t) z$. The input map $\mathcal{B}: \mathbf{L}_{2}(0, \infty ; U) \rightarrow Z$ of an input stable state linear system is defined by

$$
\mathcal{B} u:=\int_{0}^{\infty} T(s) B u(s) d s .
$$

The input and output stability properties are related to the existence of solutions to Lyapunov equations (see Grabowski [15] and Hansen and Weiss [16]).

Lemma 2.6. The state linear system $\Sigma(A, B, C, D)$ is input stable if and only if the following controllability Lyapunov equation has a bounded nonnegative solution $L \in \mathcal{L}(Z):$

$$
A L z+L A^{*} z=-B B^{*} z \quad \forall z \in D\left(A^{*}\right) .
$$

In this case, the controllability gramian $L_{B}:=\mathcal{B B}^{*}$ is the smallest bounded nonnegative solution of (2.1) and $L_{B}^{1 / 2} T(t)^{*} z \rightarrow 0$ as $t \rightarrow \infty$ for all $z \in Z$.

The state linear system $\Sigma(A, B, C, D)$ is output stable if and only if the following observability Lyapunov equation has a bounded nonnegative solution $L \in \mathcal{L}(Z)$ :

$$
A^{*} L z+L A z=-C^{*} C z \quad \forall z \in D(A) .
$$

In this case, the observability gramian $L_{C}:=\mathcal{C}^{*} \mathcal{C}$ is the smallest bounded nonnegative solution of (2.2) and $L_{C}^{1 / 2} T(t) z \rightarrow 0$ as $t \rightarrow \infty$ for all $z \in Z$.

The Hankel operator of a system is a fundamental concept.

Definition 2.7. For $\mathbf{G} \in \mathbf{L}_{\infty}((-i \infty, i \infty) ; \mathcal{L}(U, Y))$ we define the Hankel operator with symbol $\mathbf{G}$ as the operator $H_{\mathbf{G}}: \mathbf{H}_{2}(U) \rightarrow \mathbf{H}_{2}(Y)$ given by

$$
H_{\mathbf{G}} f=\Pi\left(\Lambda_{\mathbf{G}} f_{-}\right) \text {for } f \in \mathbf{H}_{2}(U),
$$

where $\Lambda_{\mathbf{G}}$ is the multiplication map on $\mathbf{L}_{2}((-i \infty, i \infty) ; U)$ induced by $\mathbf{G}, \Pi$ is the orthogonal projection from $\mathbf{L}_{2}((-i \infty, i \infty) ; U)$ onto $\mathbf{H}_{2}(U)$, and $f_{-}(s):=f(-s)$.

Given $h \in \mathbf{L}_{1}^{\text {loc }}([0, \infty) ; \mathcal{L}(U, Y))$, we define the (time-domain) Hankel operator $\Gamma_{h}$ associated with $h$ for $u \in \mathbf{L}_{2}^{\text {loc }}([0, \infty) ; U)$ with compact support by

$$
\left(\Gamma_{h}\right)(t):=\int_{0}^{\infty} h(t+\tau) u(\tau) d \tau .
$$

There is a nice relationship between the time-domain and frequency-domain Hankel operators. 
Lemma 2.8. Suppose that $\Sigma(A, B, C, 0)$ is a system-stable system with impulse response $h(t)=C T(t) B$ and transfer function $\mathbf{G}$.

1. $\Gamma_{h}=\mathcal{C B}$ and it is isomorphic to $H_{\mathbf{G}}$ via

$$
\widehat{\left(\Gamma_{h} u\right)}(i \omega)=\left(H_{\mathbf{G}} \hat{u}\right)(i \omega) \text { for } u \in \mathbf{L}_{2}([0, \infty) ; U) .
$$

Moreover,

$$
\left\|H_{\mathbf{G}}\right\|=\left\|\Gamma_{h}\right\|=r^{\frac{1}{2}}\left(L_{B} L_{C}\right),
$$

where $r$ denotes the spectral radius and $L_{B}, L_{C}$ are the controllability and observability gramians, respectively, of $\Sigma(A, B, C, 0)$.

2. If $\sigma>r^{\frac{1}{2}}\left(L_{1} L_{2}\right)$, where $L_{1}, L_{2}$ are arbitrary bounded nonnegative solutions of the Lyapunov equations (2.1), (2.2), respectively, then $N_{\sigma}:=\left(I-\frac{1}{\sigma^{2}} L_{1} L_{2}\right)^{-1} \in$ $\mathcal{L}(Z)$. Moreover, $W=N_{\sigma} L_{1}$ is nonnegative.

Proof. 1. See Oostveen [21, Lemma 7.1.5].

2. Now $\sigma^{2}>r\left(L_{1} L_{2}\right)$ implies that the spectral radius of $\frac{1}{\sigma^{2}} L_{1} L_{2}$ is less than 1 and so $I-\frac{1}{\sigma^{2}} L_{1} L_{2}$ is boundedly invertible. Noting that $L_{1} \geq 0$, the following shows that $W \geq 0$ :

$$
W=\left(I-\frac{1}{\sigma^{2}} L_{1} L_{2}\right)^{-1} L_{1}=L_{1}^{1 / 2}\left(I-\frac{1}{\sigma^{2}} L_{1}^{1 / 2} L_{2} L_{1}^{1 / 2}\right)^{-1} L_{1}^{1 / 2}
$$

3. State linear systems: Some new results. In this section we develop several new results for state linear systems that we use in what follows, many of which are interesting in their own right.

First we examine the properties of the various concepts of stability from Definition 2.3 more closely.

Lemma 3.1. If $\Sigma(A, B, C, D)$ is output stable with observability gramian $L_{C}$, then for all $u \in \mathbf{L}_{2}^{\text {loc }}(\mathbb{R} ; U)$ with compact support

$$
L_{C}^{1 / 2} \int_{-\infty}^{t} T(t-s) B u(s) d s \rightarrow 0 \quad \text { as } t \rightarrow \infty
$$

Proof. Let $\tau>0$ be such that $u(t)=0$ for $t>\tau$. Then

$$
\begin{aligned}
L_{C}^{1 / 2} \int_{-\infty}^{t} T(t-s) B u(s) d s & =L_{C}^{1 / 2} \int_{-\infty}^{\tau} T(t-\tau) T(\tau-s) B u(s) d s \\
& =L_{C}^{1 / 2} T(t-\tau) z(\tau),
\end{aligned}
$$

where $z(\tau)=\int_{-\infty}^{\tau} T(\tau-s) B u(s) d s$ is independent of $t$. Lemma 2.6 now gives the result.

We recall that the output of a state linear system $\Sigma(A, B, C, D)$ with locally square integrable inputs $u$ with support bounded to the left is defined by

$$
y(t)=\int_{-\infty}^{t} C T(t-s) B u(s) d s+D u(t) .
$$

Output stability does not imply input-output stability, but we do have the following partial result.

Lemma 3.2. If $\Sigma(A, B, C, D)$ is output stable, then for inputs $u \in \mathbf{L}_{2}^{\text {loc }}(\mathbb{R} ; U)$ with compact support, the output given by (3.1) is in $\mathbf{L}_{2}(\mathbb{R} ; Y)$. 
Proof. Since $u$ is square integrable we can assume without loss of generality that $D=0$. Since $u$ has compact support, there exists a $\tau>0$ such that $u(t)=0$ for $t>\tau$. We calculate the output $y(t)$ for $t>\tau$ as

$$
\begin{aligned}
y(t) & =\int_{-\infty}^{\tau} C T(t-\tau) T(\tau-s) B u(s) d s \\
& =C T(t-\tau) \int_{-\infty}^{\tau} T(\tau-s) B u(s) d s=C T(t-\tau) z(\tau),
\end{aligned}
$$

where

$$
z(\tau):=\int_{-\infty}^{\tau} T(\tau-s) B u(s) d s
$$

Since $\Sigma(A, B, C, D)$ is output stable we have

$$
\begin{aligned}
\int_{\tau}^{\infty}\|y(t)\|^{2} d t & =\int_{0}^{\infty}\|C T(t) z(\tau)\|^{2} d t \\
& \leq \text { const. }\|z(\tau)\|^{2}<\infty
\end{aligned}
$$

Since the output of a state linear system is always locally square integrable and the output has support bounded to the left by causality we have

$$
\int_{-\infty}^{\infty}\|y(t)\|^{2} d t=\int_{-\infty}^{\tau}\|y(t)\|^{2} d t+\int_{\tau}^{\infty}\|y(t)\|^{2} d t<\infty
$$

We next examine the connection between the transfer function and the characteristic function of a state linear system.

DEFINITION 3.3. For an output stable state linear system we define $\widehat{\mathcal{C}}: Z \rightarrow$ $\mathbf{H}_{2}(Y)$ by $\widehat{\mathcal{C}} z:=\widehat{\mathcal{C} z}$.

For an input stable state linear system we define $\widehat{\mathcal{B}}$ for $u \in U, z \in Z, s \in \mathbb{C}_{0}^{+}$by $\langle\widehat{\mathcal{B}}(s) u, z\rangle:=\left\langle u, \widehat{\mathcal{B}^{*} z}(\bar{s})\right\rangle$.

The following lemma shows that input or output stability ensures that the characteristic function and the transfer function are equal on the set where they are both defined. Parts of this lemma were shown for well-posed linear systems in [11, Lemma $2.3]$.

LEMMA 3.4.

1. If the state linear system $\Sigma(A, B, C, D)$ is output stable, then

$$
\begin{aligned}
& \mathbf{G}(s)=D+\widehat{\mathcal{C}}(s) B \quad \forall s \in \mathbb{C}_{0}^{+}, \\
& \mathbf{G}(s)=D+C(s I-A)^{-1} B=\mathfrak{G}(s) \quad \forall s \in \mathbb{C}_{0}^{+} \cap \rho(A) .
\end{aligned}
$$

Moreover, for all $u \in U$ we have that $(\mathbf{G}-D) u \in \mathbf{H}_{2}(Y)$.

2. If the state linear system $\Sigma(A, B, C, D)$ is input stable, then (3.3) holds and

$$
\mathbf{G}(s)=D+C \widehat{\mathcal{B}}(s) \quad \forall s \in \mathbb{C}_{0}^{+} .
$$

Moreover, for all $u \in U, y \in Y$ we have that $\langle(\mathbf{G}-D) u, y\rangle \in \mathbf{H}_{2}$.

Proof. 1. Taking Laplace transforms of $C T(\cdot) z$, we obtain $C(s I-A)^{-1} z=\widehat{\mathcal{C}}(s) z$ and $\mathbf{G}(s)=\widehat{\mathcal{C}}(s) B+D$ for $s$ in some right half-plane (see [4, Lemma 2.1.11]). Now since $\Sigma$ is output stable, $\hat{\mathcal{C}} z \in \mathbf{H}_{2}(Y)$ for all $z \in Z$ and so $\widehat{\mathcal{C}}$ is holomorphic in $\mathbb{C}_{0}^{+}$and 
(3.2) holds. Again using that $\widehat{\mathcal{C}}$ is holomorphic on $\mathbb{C}_{0}^{+}$, the equality $\widehat{\mathcal{C}}(s)(s I-A)=C$ for $s$ in some right half-plane extends to $\mathbb{C}_{0}^{+}$. Thus for $s \in \mathbb{C}_{0}^{+} \cap \rho(A)$ we have

$$
\hat{\mathcal{C}}(s)(s I-A)(s I-A)^{-1} B=C(s I-A)^{-1} B,
$$

which proves (3.3).

2. Similarly, input stability implies that $V(s) z \in \mathbf{H}_{2}(U)$ for $z \in Z$, where $V(s) z:=B^{*}\left(s I-A^{*}\right)^{-1} z$ on some right half-plane. Then $\langle u, V(\bar{s}) z\rangle=\langle\widehat{\mathcal{B}}(s) u, z\rangle$ for all $z \in Z, u \in U$. So for all $s$ in some right half-plane we have

$$
\left\langle(s I-A)^{-1} B u, z\right\rangle=\langle\widehat{\mathcal{B}}(s) u, z\rangle,
$$

and letting $z=C^{*} x$, we obtain $\mathbf{G}=C \widehat{\mathcal{B}}+D$ on some right half-plane. Using that $\widehat{\mathcal{B}}$ is holomorphic, we obtain (3.4). To show (3.3) choose $z \in D\left(A^{*}\right)$, let $x=\left(\bar{s} I-A^{*}\right) z$, and substitute in (3.5) to obtain for $s$ in some right half-plane

$$
\langle B u, x\rangle=\langle(s I-A) \widehat{\mathcal{B}}(s) u, x\rangle .
$$

This extends to $s \in \mathbb{C}_{0}^{+}$since $\widehat{\mathcal{B}}$ is holomorphic and to all $x \in Z$ by continuity. Thus

$$
(s I-A) \widehat{\mathcal{B}}(s)=B \quad \forall s \in \mathbb{C}_{0}^{+},
$$

which proves (3.3).

It turns out that the existence of boundary functions of $\mathbf{H}_{2}$ functions is crucial in our later proofs. We recall some basic results from [25]. Let $\omega \in \mathbb{R}$ and consider for $\alpha>0$ the cone

$$
\Gamma_{\alpha}=\left\{s \in \mathbb{C}_{0}^{+}:|\operatorname{Im}(s)-\omega|<\alpha \operatorname{Re}(s)\right\} .
$$

If $f \in \mathbf{H}_{2}(H)$, where $H$ is a separable Hilbert space, then for almost all $\omega \in \mathbb{R}$ and all $\alpha>0$ the limit

$$
\lim _{s \rightarrow i \omega, s \in \Gamma_{\alpha}} f(s)
$$

exists. Such a limit is called a nontangential limit, and it associates with an $\mathbf{H}_{2}(H)$ function a function in $\mathbf{L}_{2}(i \mathbb{R}, H)$ (see [25, Theorems 4.6.B and 4.8.B]).

It is well known that if $U$ and $Y$ are finite-dimensional and for all $u \in U$ we have $\mathbf{G} u \in \mathbf{H}_{2}(Y)$, then $\mathbf{G} \in \mathbf{H}_{2}(\mathcal{L}(U, Y))$. Hence we obtain an almost everywhere defined boundary function $\mathbf{G}: i \mathbb{R} \rightarrow \mathcal{L}(U, Y)$.

In the case where $U$ and $Y$ are infinite-dimensional we do have that for all $u \in U$ the function $\mathbf{G}(\cdot) u$ has a boundary function in $\mathbf{L}_{2}(i \mathbb{R} ; Y)$. However, in general there does not exist an almost everywhere defined function $F: i \mathbb{R} \rightarrow \mathcal{L}(U, Y)$ such that $F(i \omega) u$ equals this boundary function (see Mikkola [19, Example 3.3.6] for a counterexample).

If $\Sigma(A, B, C, D)$ is input or output stable and $\sigma(A) \cap i \mathbb{R}$ has measure zero, then $\mathbf{G}(s)=\mathfrak{G}(s)$ on $\mathbb{C}_{0}^{+} \cap \rho(A)$ by Lemma 3.4 and since $\mathfrak{G}(s) \rightarrow \mathfrak{G}(i \omega)$ as $s \rightarrow i \omega$ by continuity of the map $s \mapsto(s I-A)^{-1}$, we have $\mathbf{G}(s) \rightarrow \mathfrak{G}(i \omega)$. So if $\Sigma(A, B, C, D)$ is input or output stable and $\sigma(A) \cap i \mathbb{R}$ has measure zero, then $\mathbf{G}$ has an almost everywhere defined operator-valued boundary function. From the above we obtain the following.

LEMma 3.5. Let $\Sigma(A, B, C, D)$ be input or output stable and assume that either $\sigma(A) \cap i \mathbb{R}$ has measure zero or $U$ and $Y$ are finite-dimensional. Then there exists an 
almost everywhere defined function $\mathbf{G}_{0}: i \mathbb{R} \rightarrow \mathcal{L}(U, Y)$ such that for almost all $\omega \in \mathbb{R}$ and all nontangential paths we have

$$
\mathbf{G}_{0}(i \omega)=\lim _{s \rightarrow i \omega} \mathbf{G}(s) .
$$

Moreover, if $i \omega \in \rho(A)$, then $\mathbf{G}_{0}(i \omega)=\mathfrak{G}(i \omega)$.

Proof. This follows from the paragraphs preceding the lemma.

We prove the following lemma that will be useful later.

Lemma 3.6. Let $f: \mathbb{C}_{0}^{+} \rightarrow \mathcal{L}(U, Y)$ be such that for every $u \in U$ we have $f(\cdot) u \in \mathbf{H}_{2}(Y)$. Assume there exists a function $f_{0} \in \mathbf{L}_{\infty}(i \mathbb{R} ; \mathcal{L}(U, Y))$ such that for all $u \in U$ there exists a set $\mathcal{N}_{u}$ of measure zero such that for all $\omega \in \mathbb{R}-\mathcal{N}_{u}$ and all nontangential paths we have

$$
f_{0}(i \omega) u=\lim _{s \rightarrow i \omega} f(s) u
$$

Then $f \in \mathbf{H}_{\infty}(\mathcal{L}(U, Y))$.

Proof. Since $f(\cdot) u \in \mathbf{H}_{2}(Y)$ we have the Poisson representation [25, Theorem 4.8.A]

$$
f(a+i b) u=\frac{1}{\pi} \int_{\mathbb{R}} \frac{b f_{0}(i \omega) u}{(t-a)^{2}+b^{2}} d t,
$$

so we have (using that the Poisson kernel has integral one)

$$
\|f(a+i b) u\| \leq \frac{1}{\pi} \int_{\mathbb{R}}\left\|f_{0}(i \omega) u\right\| \frac{b}{(t-a)^{2}+b^{2}} d t \leq \operatorname{ess} \sup _{t \in \mathbb{R}}\left\|f_{0}(i t)\right\|\|u\| .
$$

This shows that

$$
\sup _{s \in \mathbb{C}_{0}^{+}}\|f(s)\| \leq \operatorname{ess} \sup _{t \in \mathbb{R}}\left\|f_{0}(i t)\right\|
$$

and since $f$ is holomorphic we have $f \in \mathbf{H}_{\infty}(\mathcal{L}(U, Y))$.

Lemma 3.7. Let $\Sigma(A, B, C, D)$ be output stable and assume that either $\sigma(A) \cap i \mathbb{R}$ has measure zero or $U$ and $Y$ are finite-dimensional. Then for inputs with compact support we have for almost all $\omega \in \mathbb{R}$

$$
\hat{y}(i \omega)=\mathbf{G}(i \omega) \hat{u}(i \omega) .
$$

Proof. We first prove the statement for the case that $u$ is zero for negative time. Now on some right half-plane we have

$$
\hat{y}(s)=\mathbf{G}(s) \hat{u}(s) .
$$

Since $\hat{y} \in \mathbf{H}_{2}(Y)$ by Lemma 3.2 (and causality) and $\mathbf{G}$ is holomorphic on $\mathbb{C}_{0}^{+}$by Lemma 3.4 this extends to $\mathbb{C}_{0}^{+}$. By Lemma 3.5 we have $\mathbf{G}(s) \rightarrow \mathbf{G}(i \omega)$ in the operator norm as $s \rightarrow i \omega$. Since $\hat{u} \in \mathbf{H}_{2}(U)$ and $\hat{y} \in \mathbf{H}_{2}(Y)$, they converge to their boundary functions as $s \rightarrow i \omega$ so we obtain (3.7). The general case follows by applying the above to the function $\underline{u}(t):=u(t-\tau)$ with output $\underline{y}(t):=y(t-\tau)$, where $y$ is the output corresponding to $u$ and $\tau$ is chosen such that $\underline{u}$ is zero for negative time.

In the proof of Lemma 3.8 we need to study systems defined on the positive time axis only and with a given initial state. We summarize some known results for this 
type of system. For an input $u \in \mathbf{L}_{2}^{\text {loc }}(0, \infty ; U)$ and initial state $x_{0} \in X$ the state $x(t) \in X$ at time $t \geq 0$ is defined by

$$
x(t)=T(t) x_{0}+\int_{0}^{t} T(t-s) B u(s) d s .
$$

If $u$ is continuously differentiable and $x_{0} \in D(A)$, then $x$ as defined above is differentiable and satisfies

$$
\dot{x}(t)=A x(t)+B u(t), \quad x(0)=x_{0} .
$$

The output of the state linear system is defined by

$$
y(t)=C x(t)+D u(t) .
$$

A state linear system is well-posed in the sense that for all $t>0$ there exists a $K>0$ such that for all $u \in \mathbf{L}_{2}^{\text {loc }}(0, \infty ; U)$ and all $x_{0} \in X$

$$
\|x(t)\|^{2}+\int_{0}^{t}\|y(s)\|^{2} d s \leq K\left(\left\|x_{0}\right\|^{2}+\int_{0}^{t}\|u(s)\|^{2} d s\right) ;
$$

i.e., the map from the initial state and the input restricted to $(0, t)$ to the state at time $t$ and the output restricted to $(0, t)$ is continuous from $X \times L^{2}(0, t ; U)$ to $X \times L^{2}(0, t ; Y)$.

Lemma 3.8. Let $\Sigma(A, B, C, 0)$ be output stable with observability gramian $L_{C}$ and let $u_{i} \in \mathbf{L}_{2}^{\text {loc }}(\mathbb{R} ; U)$ be an input with compact support. Denote by $y_{i}$ the output of $\Sigma(A, B, C, 0)$ with input $u_{i}$ given by (3.1) and by $y_{i}^{L C}$ the output of $\Sigma\left(A, B, B^{*} L_{C}, 0\right)$ with input $u_{i}$ given by the corresponding (3.1). Then we have the following:

$$
\int_{-\infty}^{\infty}\left\langle y_{1}(t), y_{2}(t)\right\rangle d t=\int_{-\infty}^{\infty}\left\langle u_{1}(t), y_{2}^{L C}(t)\right\rangle d t+\int_{-\infty}^{\infty}\left\langle y_{1}^{L C}(t), u_{2}(t)\right\rangle d t
$$

Proof. We first note that the integrals in (3.9) are well defined since $y_{i} \in \mathbf{L}_{2}(\mathbb{R} ; Y)$ by Lemma 3.2 and the $u_{i}$ have compact support. Set $z_{i}(t)=\int_{-\infty}^{t} T(t-s) B u_{i}(s) d s$ for $i=1,2$. If $u_{i}$ is continuously differentiable, then $z_{i}(t)$ is differentiable and

$$
\begin{aligned}
& \frac{d}{d t}\left\langle z_{1}(t), L_{C} z_{2}(t)\right\rangle \\
& =\left\langle A z_{1}(t)+B u_{1}(t), L_{C} z_{2}(t)\right\rangle+\left\langle L_{C} z_{1}(t), A z_{2}(t)+B u_{2}(t)\right\rangle \\
& =\left\langle B u_{1}(t), L_{C} z_{2}(t)\right\rangle+\left\langle L_{C} z_{1}(t), B u_{2}(t)\right\rangle-\left\langle C z_{1}(t), C z_{2}(t)\right\rangle,
\end{aligned}
$$

where we have used (2.2). On integrating the above we obtain

$$
\begin{aligned}
\left\langle z_{1}(t), L_{C} z_{2}(t)\right\rangle & \\
= & \int_{-\infty}^{t}\left\langle u_{1}(s), B^{*} L_{C} z_{2}(s)\right\rangle d s \\
& +\int_{-\infty}^{t}\left\langle B^{*} L_{C} z_{1}(s), u_{2}(s)\right\rangle d s-\int_{-\infty}^{t}\left\langle C z_{1}(s), C z_{2}(s)\right\rangle d s . \\
= & \int_{-\infty}^{t}\left\langle u_{1}(s), y_{2}^{L C}(s)\right\rangle d s \\
& +\int_{-\infty}^{t}\left\langle y_{1}^{L C}(s), u_{2}(s)\right\rangle d s-\int_{-\infty}^{t}\left\langle y_{1}(s), y_{2}(s)\right\rangle d s .
\end{aligned}
$$


From Lemma 3.1 we conclude that the left-hand side of (3.12) converges to zero as $t \rightarrow \infty$. This proves (3.9) for the case of continuously differentiable inputs, and the general case follows by the following approximation argument. Let $u_{i} \in \mathbf{L}_{2}^{\text {loc }}(\mathbb{R} ; U)$ be inputs with compact support and let $u_{i}^{n} \in \mathbf{L}_{2}^{\text {loc }}(\mathbb{R} ; U)$ be continuously differentiable inputs with compact support that converge to $u_{i}$ in $\mathbf{L}_{2}(\mathbb{R} ; U)$. Let $\tau$ be such that $u_{i}$ and $u_{i}^{n}$ are equal to zero on $(\tau, \infty)$, and assume that $u_{i}$ and $u_{i}^{n}$ are zero on $(-\infty, 0)$. By the well-posedness there exists a $K(\tau)$ such that

$$
\int_{-\infty}^{\tau}\left\|y_{i}(s)-y_{i}^{n}(s)\right\|^{2} d s \leq K(\tau) \int_{-\infty}^{\tau}\left\|u_{i}(s)-u_{i}^{n}(s)\right\|^{2} d s
$$

and hence

$$
\int_{-\infty}^{\tau}\left\|y_{i}(s)-y_{i}^{n}(s)\right\|^{2} d s \rightarrow 0 \text { as } n \rightarrow \infty
$$

For $z_{i}^{n}(\tau):=\int_{-\infty}^{\tau} T(\tau-s) B u_{i}(s) d s$ we have

$$
\int_{\tau}^{\infty}\left\|y_{i}(s)-y_{i}^{n}(s)\right\|^{2} d s=\int_{0}^{\infty}\left\|C T(s)\left(z_{i}(\tau)-z_{i}^{n}(\tau)\right)\right\|^{2} d s .
$$

Since $\Sigma(A, B, C, 0)$ is output stable, there exists a $\gamma>0$ such that

$$
\int_{\tau}^{\infty}\left\|y_{i}(s)-y_{i}^{n}(s)\right\|^{2} d s \leq \gamma\left\|z_{i}(\tau)-z_{i}^{n}(\tau)\right\|^{2},
$$

and by the well-posedness there exists a $K(\tau)$ such that

$$
\left\|z_{i}(\tau)-z_{i}^{n}(\tau)\right\|^{2} \leq K(\tau) \int_{-\infty}^{\tau}\left\|u_{i}(s)-u_{i}^{n}(s)\right\|^{2} d s
$$

Hence

$$
\int_{\tau}^{\infty}\left\|y_{i}(s)-y_{i}^{n}(s)\right\|^{2} d s \rightarrow 0 \text { as } n \rightarrow \infty
$$

So we have $y_{i}^{n} \rightarrow y_{i}$ in $\mathbf{L}_{2}(\mathbb{R} ; Y)$. By the compact support of the inputs $u_{i}$ and $u_{i}^{n}$ we need only $y_{i}^{n, L C} \rightarrow y_{i}^{L C}$ in $\mathbf{L}_{2}(-\infty, \tau ; Y)$ to establish (3.9). This convergence follows from the well-posedness as above. Using this (3.9) follows. The case that $u_{i}$ is not zero on $(-\infty, 0)$ can be reduced to the case that this is the case by a time-shift as in the proof of Lemma 3.7 .

We also need to study anticausal outputs of state linear systems. The anticausal output of the state linear system $\Sigma(A, B, C, D)$ for an input $u \in \mathbf{L}_{2}^{\text {loc }}(\mathbb{R} ; U)$ with support bounded to the right is defined as

$$
y^{a}(t):=\int_{t}^{\infty} C T(s-t) B u(s) d s+D u(t) .
$$

We have the following analogue of Lemma 3.2.

Lemma 3.9. If $\Sigma(A, B, C, D)$ is output stable, then for inputs $u \in \mathbf{L}_{2}^{\text {loc }}(\mathbb{R} ; U)$ with compact support, the anticausal output given by (3.13) is in $\mathbf{L}_{2}(\mathbb{R} ; Y)$.

Proof. The proof is as in the proof of Lemma 3.2.

We have the following analogue of Lemma 3.7. 
Lemma 3.10. Let $\Sigma(A, B, C, D)$ be output stable and assume that either $\sigma(A) \cap i \mathbb{R}$ has measure zero or $U$ and $Y$ are finite-dimensional. Then for inputs with compact support we have for almost all $\omega \in \mathbb{R}$

$$
\hat{y}^{a}(i \omega)=\mathbf{G}(-i \omega) \hat{u}(i \omega),
$$

where $y^{a}$ is the anticausal output of $\Sigma(A, B, C, D)$ defined by (3.13).

Proof. This follows as in the proof of Lemma 3.7, but now by first assuming $u$ to be zero for positive time and using the Hardy space $\mathbf{H}_{2}$ over the left half-plane. Details are as follows. We first prove the statement for the case that $u$ is zero for positive time. Now on some left half-plane we have

$$
\hat{y}^{a}(s)=\mathbf{G}(-s) \hat{u}(s) .
$$

Since $\hat{y}^{a} \in \mathbf{H}_{2}\left(\mathbb{C}_{0}^{-} ; Y\right)$ by Lemma 3.9 (and anticausality) and $\mathbf{G}$ is holomorphic on $\mathbb{C}_{0}^{+}$by Lemma 3.4 this extends to $\mathbb{C}_{0}^{-}$. By Lemma 3.5 we have $\mathbf{G}(s) \rightarrow \mathbf{G}(i \omega)$ in the operator norm as $s \rightarrow i \omega$. Since $\hat{u} \in \mathbf{H}_{2}\left(\mathbb{C}_{0}^{-} ; U\right)$ and $\hat{y}^{a} \in \mathbf{H}_{2}\left(\mathbb{C}_{0}^{-} ; Y\right)$, they converge to their boundary functions as $s \rightarrow i \omega$ so we obtain (3.14). The general case follows by applying the above to the function $\underline{u}(t):=u(t+\tau)$ with output $y^{a}(t):=y^{a}(t+\tau)$, where $y$ is the output corresponding to $u$ and $\tau$ is chosen such that $\underline{u}$ is zero for positive time.

The next result is a consequence of Lemma 3.8.

Lemma 3.11. Let $\Sigma(A, B, C, 0)$ be input and output stable with observability gramian $L_{C}$ and let $u_{i} \in \mathbf{L}_{2}^{\text {loc }}(\mathbb{R} ; U)$ be an input with compact support. Denote by $y_{i}$ the output of $\Sigma(A, B, C, 0)$ with input $u_{i}$ given by (3.1) and by $y_{i}^{a}$ the anticausal output of $\Sigma\left(A^{*}, L_{C} B, B^{*}, 0\right)$ with input $u_{i}$ given by the corresponding (3.13). Then we have $y_{i}^{a} \in \mathbf{L}_{2}(\mathbb{R} ; U)$ and the following:

$$
\int_{-\infty}^{\infty}\left\langle y_{1}(t), y_{2}(t)\right\rangle d t=\int_{-\infty}^{\infty}\left\langle y_{1}^{a}(t), u_{2}(t)\right\rangle d t+\int_{-\infty}^{\infty}\left\langle u_{1}(t), y_{2}^{a}(t)\right\rangle d t .
$$

Proof. Since $\Sigma(A, B, C, 0)$ is input stable the system $\Sigma\left(A^{*}, L_{C} B, B^{*}, 0\right)$ is output stable. Lemma 3.9 then implies that $y_{i}^{a} \in \mathbf{L}_{2}(\mathbb{R} ; U)$. Equation (3.16) follows from (3.9) by an application of Fubini's theorem and a change of variables.

Lemma 3.12. If $\Sigma(A, B, C, 0)$ is input and output stable with the observability gramian $L_{C}$, then

$$
\begin{aligned}
& \left\langle L_{C} B u, \widehat{\mathcal{B}}(s) u\right\rangle+\left\langle\widehat{\mathcal{B}}(s) u, L_{C} B u\right\rangle \\
& =\|\mathbf{G}(s) u\|^{2}+2 \operatorname{Re} s\left\|L_{C}^{1 / 2} \widehat{\mathcal{B}}(s) u\right\|^{2} \quad \forall s \in \mathbb{C}_{0}^{+}, u \in U .
\end{aligned}
$$

Proof. We obtain a straightforward frequency domain identity from the Lyapunov equation (2.2):

$$
\left(\bar{s} I-A^{*}\right) L_{C}+L_{C}(s I-A)=C^{*} C+2 \operatorname{Re} s L_{C} .
$$

This leads to the following identity on some right half-plane:

$$
\begin{aligned}
& B^{*} L_{C}(s I-A)^{-1} B+B^{*}\left(\bar{s} I-A^{*}\right)^{-1} L_{C} B \\
& =B^{*}\left(\bar{s} I-A^{*}\right)^{-1} C^{*} C(s I-A)^{-1} B+2 \operatorname{Re} s B^{*}(\bar{s} I-A)^{-1} L_{C}(s I-A)^{-1} B .
\end{aligned}
$$

From this we obtain (3.17) for $s$ in some right half-plane using Lemma 3.4. From the input stability of $\Sigma(A, B, C, D)$ we obtain that $\widehat{\mathcal{B}}$ and $\mathbf{G}$ are holomorphic on $\mathbb{C}_{0}^{+}$. 
From the appendix (Corollaries 9.1 and 9.4) it follows that all terms in (3.17) are real-analytic on $\mathbb{C}_{0}^{+}$. By the identity theorem for real-analytic functions we obtain (3.17) for $s \in \mathbb{C}_{0}^{+}$.

The next lemma is the main result of this section.

LEMma 3.13. Let $\Sigma(A, B, C, 0)$ be input and output stable with the observability gramian $L_{C}$ and assume that either $\sigma(A) \cap i \mathbb{R}$ has measure zero or $U$ and $Y$ are finite-dimensional. Then for almost all $\omega \in \mathbb{R}$, all $u \in U$, and all nontangential paths

$$
\lim _{s \rightarrow i \omega} \operatorname{Re} s\left\|L_{C}^{1 / 2} \widehat{\mathcal{B}}(s) u\right\|^{2}=0 .
$$

Proof. Let $u_{i}$ be locally square integrable with compact support. Let $y_{i}$ denote the output of $\Sigma(A, B, C, 0)$ for input $u_{i}$ from (3.1) and let $y_{i}^{a}$ denote the anticausal output of $\Sigma\left(A^{*}, L_{C} B, B^{*}, 0\right)$ for input $u_{i}$ from the corresponding (3.13). By Fourier transforming (3.16) we obtain

$$
\int_{-\infty}^{\infty}\left\langle\hat{y}_{1}(i \omega), \hat{y}_{2}(i \omega)\right\rangle d \omega=\int_{-\infty}^{\infty}\left\langle\hat{y}_{1}^{a}(i \omega), \hat{u}_{2}(i \omega)\right\rangle d \omega+\int_{-\infty}^{\infty}\left\langle\hat{u}_{1}(i \omega), \hat{y}_{2}^{a}(i \omega)\right\rangle d \omega .
$$

From Lemmas 3.7 and 3.10 we obtain

$$
\begin{gathered}
\int_{-\infty}^{\infty}\left\langle\mathbf{G}(i \omega) \hat{u}_{1}(i \omega), \mathbf{G}(i \omega) \hat{u}_{2}(i \omega)\right\rangle d \omega \\
=\int_{-\infty}^{\infty}\left\langle\mathbf{G}^{L C}(-i \omega) \hat{u}_{1}(i \omega), \hat{u}_{2}(i \omega)\right\rangle d \omega+\int_{-\infty}^{\infty}\left\langle\hat{u}_{1}(i \omega), \mathbf{G}^{L C}(-i \omega) \hat{u}_{2}(i \omega)\right\rangle d \omega
\end{gathered}
$$

where $\mathbf{G}^{L C}$ is the transfer function of the system $\Sigma\left(A^{*}, L_{C} B, B^{*}, 0\right)$. Letting $u_{i}(t)=$ $f(t) v$, where $f$ is a scalar function with compact support and $v \in U$, we obtain

$$
\int_{-\infty}^{\infty}|\hat{f}(i \omega)|^{2}\left(\left\langle\mathbf{G}^{L C}(-i \omega) v, v\right\rangle+\left\langle v, \mathbf{G}^{L C}(-i \omega) v\right\rangle-\|\mathbf{G}(i \omega) v\|^{2}\right) d \omega=0 .
$$

From (3.17) we obtain using $\mathbf{G}^{L C}(s)=\widehat{\mathcal{B}}(\bar{s})^{*} L_{C} B$ that for all $s \in \mathbb{C}_{0}^{+}$

$$
\left\langle\mathbf{G}^{L C}(\bar{s}) v, v\right\rangle+\left\langle v, \mathbf{G}^{L C}(\bar{s}) v\right\rangle-\|\mathbf{G}(s) v\|^{2}=2 \operatorname{Re} s\left\|L_{C}^{1 / 2} \widehat{\mathcal{B}}(s) v\right\|^{2} \geq 0 .
$$

Taking nontangential limits (which exist for all three terms on the left-hand side) we obtain for almost all $\omega \in \mathbb{R}$

$$
\left\langle\mathbf{G}^{L C}(-i \omega) v, v\right\rangle+\left\langle v, \mathbf{G}^{L C}(-i \omega) v\right\rangle-\|\mathbf{G}(i \omega) v\|^{2} \geq 0 .
$$

Combining (3.19) and (3.20) we obtain for almost all $\omega \in \mathbb{R}$

$$
|\hat{f}(i \omega)|^{2}\left(\left\langle\mathbf{G}^{L C}(-i \omega) v, v\right\rangle+\left\langle v, \mathbf{G}^{L C}(-i \omega) v\right\rangle-\|\mathbf{G}(i \omega) v\|^{2}\right)=0 .
$$

Now let $f: \mathbb{R} \rightarrow \mathbb{C}$ be a function that has compact support and such that $\hat{f}(i \omega) \neq 0$ for almost all $\omega \in \mathbb{R}$ (for example, the function equal to 1 on $[0,1]$ and zero elsewhere). Then we obtain for all $v \in U$ and almost all $\omega \in \mathbb{R}$

$$
\left\langle\mathbf{G}^{L C}(-i \omega) v, v\right\rangle+\left\langle v, \mathbf{G}^{L C}(-i \omega) v\right\rangle-\|\mathbf{G}(i \omega) v\|^{2}=0,
$$

and comparing the above with (3.17) proves (3.18). 
It is an interesting open question whether Lemma 3.13 is true for infinitedimensional $U$ and $Y$ without the spectrum assumption.

We need the following property of the system $\Sigma\left(A, B, B^{*} L_{C}, 0\right)$ shown in Weiss and Weiss [31, Theorem 11.1] (see also Oostveen [21, Lemma 4.2.6]).

LEMMA 3.14. If $\Sigma(A, B, C, D)$ is output stable and input-output stable with observability gramian $L_{C}$, then $\Sigma\left(A,-, B^{*} L_{C},-\right)$ is output stable.

By duality we obtain the following.

Corollary 3.15. If $\Sigma(A, B, C, D)$ is input stable and input-output stable with controllability gramian $L_{B}$, then $\Sigma\left(A, L_{B} C^{*},-,-\right)$ is input stable.

Lemma 3.13 has an easy corollary.

COROllary 3.16. Let $\Sigma(A, B, C, 0)$ be a system-stable state linear system and assume that either $\sigma(A) \cap i \mathbb{R}$ has measure zero or $U$ and $Y$ are finite-dimensional. Denote by $\widehat{\mathcal{B}}_{L B}(\bar{s})^{*}$ and $\widehat{\mathcal{C}}_{L C}(s)$ the holomorphic extensions to $\mathbb{C}_{0}^{+}$of $C L_{B}\left(s I-A^{*}\right)^{-1}$ and $B^{*} L_{C}(s I-A)^{-1}$, respectively. Then for almost all $\omega \in \mathbb{R}$, all $u \in U$, and all nontangential paths

$$
\begin{aligned}
& \lim _{s \rightarrow i \omega} \operatorname{Re} s\left\|L_{C}^{1 / 2} \widehat{\mathcal{B}}_{L B}(s) u\right\|^{2}=0, \\
& \lim _{s \rightarrow i \omega} \operatorname{Re} s\left\|L_{B}^{1 / 2} \widehat{\mathcal{C}}(\bar{s})^{*} y\right\|^{2}=0 \\
& \lim _{s \rightarrow i \omega} \operatorname{Re} s\left\|L_{B}^{1 / 2} \widehat{\mathcal{C}}_{L C}(\bar{s})^{*} y\right\|^{2}=0 .
\end{aligned}
$$

Proof. Equation (3.21) follows from Lemma 3.13, since by Lemma 3.15, $\Sigma\left(A, L_{B} C^{*}\right.$, $C, 0)$ is input stable and output stable and has observability gramian $L_{C}$. Equation (3.22) is the dual of (3.18), and (3.23) is the dual of (3.21).

4. Riccati equations. In this section we obtain some new results on stabilizability and Riccati equations for state linear systems that we will need in what follows. First we introduce concepts of stabilizability from Curtain [10] that are refinements of the definitions introduced in Curtain and Oostveen [7].

Definition 4.1. $\Sigma(A, B, C, D)$ is output stabilizable if there exists an $F \in$ $\mathcal{L}(Z, U)$ such that $\Sigma(A+B F, B,[C ; F], 0)$ is output stable. $\Sigma(A, B, C, D)$ is input stabilizable if there exists an $L \in \mathcal{L}(Y, Z)$ such that $\Sigma(A+L C,[B, L], C, 0)$ is input stable.

The following are extensions of the results in Curtain and Oostveen [7]. In fact, they are special cases of analogous results for the very large class of well-posed linear systems in Mikkola [19]. Since the proofs there are not so accessible, we give simple proofs here.

THEOREM 4.2. If the state linear system $\Sigma(A, B, C, 0)$ is output stabilizable, then there exists a smallest bounded nonnegative solution of the control Riccati equation for $z \in D(A)$ :

$$
A^{*} Q z+Q A z+C^{*} C z-Q B B^{*} Q z=0 .
$$

Moreover, for any bounded nonnegative solution, $\Sigma\left(A_{Q}, B,\left[C ;-B^{*} Q\right], 0\right)$ is output stable, where $A_{Q}=A-B B^{*} Q$. If, in addition, $\Sigma(A, B, C, 0)$ is input stabilizable, then it is system-stable. If $\Sigma(A, B, C, 0)$ is input stabilizable, then there exists a smallest bounded nonnegative solution to the filter Riccati equation for $z \in D\left(A^{*}\right)$ :

$$
A P z+P A^{*} z-P C^{*} C P z+B B^{*} z=0 .
$$


Moreover, for any bounded nonnegative solution, $\Sigma\left(A_{P},\left[B,-P C^{*}\right], C, 0\right)$ is input stable, where $A_{P}=A-P C^{*} C$. If, in addition, $\Sigma(A, B, C, 0)$ is output stabilizable, then it is system-stable.

Proof. The existence of a smallest bounded nonnegative solution to the Riccati equation has been shown in Curtain and Oostveen [7]. In fact, it follows from [4, Theorem 6.2.4], since output stabilizability implies optimizability. Next we note that the output stability of $\Sigma\left(A_{Q}, B,\left[C ;-B^{*} Q\right], 0\right)$ follows from the following equivalent formulation of the Riccati equation:

$$
A_{Q}^{*} Q z+Q A_{Q} z+Q B B^{*} Q z+C^{*} C z=0 \text { for } z \in D(A) .
$$

This is the observability Lyapunov equation for $\Sigma\left(A_{Q}, B,\left[C ;-B^{*} Q\right], 0\right)$, and Lemma 2.6 shows that it is output stable.

Next we observe that the input stabilizability guarantees the existence of a solution $P$ to the dual filter Riccati equation (4.2). This in turn shows that the solutions to the Lyapunov equations of the system $\Sigma\left(A_{Q}, B,\left[C ;-B^{*} Q\right], 0\right)$ are $Q$ and $P(I+Q P)^{-1}$ (we use the dual version of Lemma 9.4.10 in [4]). So this system is input stable (see Lemma 2.6). So, from Theorem 3.4, we can write the transfer function $[\mathbf{N} ; \mathbf{M}]$ of the closed-loop system on $\mathbb{C}_{0}^{+}$in two ways:

$$
[\mathbf{N} ; \mathbf{M}]-[0 ; I]=\widehat{\mathcal{C}}_{Q} B=\left[C ;-B^{*} Q\right] \widehat{\mathcal{B}}_{Q},
$$

where $\widehat{\mathcal{C}}_{Q} z$ is the Laplace transform of $\left[C ;-B^{*} Q\right] T_{Q}(t) z,\left\langle\widehat{\mathcal{B}}_{Q} u, z\right\rangle$ is the Laplace transform of $\left\langle T_{Q}(t) B u, z\right\rangle$ for all $z \in Z$ and $u \in U$, and $T_{Q}$ is the semigroup generated by $A_{Q}$. We use this latter version of $[\mathbf{N} ; \mathbf{M}]$ to compute for $s \in \mathbb{C}_{0}^{+}$

$$
\begin{aligned}
& {[\mathbf{N}(s) ; \mathbf{M}(s)]^{*}[\mathbf{N}(s) ; \mathbf{M}(s)]} \\
& =\widehat{\mathcal{B}}_{Q}^{*}(s)\left[C^{*} C+Q B B^{*} Q\right] \widehat{\mathcal{B}}_{Q}(s)+I-\widehat{\mathcal{B}}_{Q}^{*}(s) Q B-B^{*} Q \widehat{\mathcal{B}}_{Q}(s) .
\end{aligned}
$$

We then use the formulation (4.3) of the Riccati equation to obtain

$$
C^{*} C+Q B B^{*} Q=A_{Q}^{*} Q+Q A_{Q}=\left(s I-A_{Q}\right)^{*} Q+Q\left(s I-A_{Q}\right)-2 \operatorname{Re} s Q .
$$

We substitute this into (4.4) and use the equality (3.6) applied to the closed-loop system $\Sigma\left(A_{Q}, B,\left[C ;-B^{*} Q\right], 0\right)$,

$$
\left(s-A_{Q}\right) \widehat{\mathcal{B}}_{Q}(s)=B \text { for } s \in \mathbb{C}_{0}^{+},
$$

to obtain

$$
[\mathbf{N}(s) ; \mathbf{M}(s)]^{*}[\mathbf{N}(s) ; \mathbf{M}(s)]=I-2 \operatorname{Re} s \widehat{\mathcal{B}}_{Q}^{*}(s) Q \widehat{\mathcal{B}}_{Q}(s) .
$$

This shows that $[\mathbf{N}(s) ; \mathbf{M}(s)]^{*}[\mathbf{N}(s) ; \mathbf{M}(s)] \leq I$ for all $s \in \mathbb{C}_{0}^{+}$. Thus $[\mathbf{N} ; \mathbf{M}] \in$ $\mathbf{H}_{\infty}(\mathcal{L}(U, Y \oplus U))$.

We proceed to deduce some interesting properties of the spectrum of the closedloop generators $A_{Q}$ and $A_{P}$ on the right half-plane.

LEMma 4.3. Suppose that the state linear system $\Sigma(A, B, C, 0)$ is input and output stabilizable. Then for any bounded nonnegative solutions $Q, P$ to the Riccati equations (4.1), (4.2), respectively, the closed-loop generators have the following properties:

1. The closed-loop operators $A_{Q}=A-B B^{*} Q$ and $A_{P}=A-P C^{*} C$ have the same spectrum and

$$
(I+P Q) A_{Q} z=A_{P}(I+P Q) z \text { for } z \in D(A) .
$$


2. Let $Q_{1}, Q_{2}$ be two bounded nonnegative solutions of (4.1). Then $\sigma\left(A_{Q_{1}}\right)=$ $\sigma\left(A_{Q_{2}}\right)$.

3. The spectra of the closed-loop generators $A_{Q}$ and $A_{P}$ in the closed right halfplane are contained in the spectrum of $A$.

Proof. 1. First we prove (4.6). As in Curtain and Zwart [4, Lemma 4.1.24] we have

$$
Q: D(A) \rightarrow D\left(A^{*}\right), \quad P: D\left(A^{*}\right) \rightarrow D(A) .
$$

So using (4.3) we obtain for $z \in D(A)$

$$
\begin{aligned}
& (I+P Q) A_{Q} z \\
& =A_{Q} z-P\left(A_{Q}^{*} Q z+Q B^{*} B Q+C C\right) z \\
& =\left(A-P C^{*} C\right) z-B B^{*} Q z-P A^{*} Q z \\
& =A_{P} z-P\left(A_{P}^{*}+C^{*} C P\right) Q z-B B^{*} Q z \\
& =A_{P} z+\left(A_{P} P+B B^{*}\right) Q z-B B Q z \text { from }(4.2) \\
& =A_{P}(I+P Q) z
\end{aligned}
$$

Since $P, Q$ are bounded nonnegative operators, $(I+P Q)$ is boundedly invertible and $\sigma\left(A_{Q}\right)=\sigma\left(A_{P}\right)$.

2. From part 1 it follows that $\sigma\left(A_{Q_{1}}\right)=\sigma\left(A_{P}\right)=\sigma\left(A_{Q_{2}}\right)$.

3. Suppose that $\lambda \in \overline{\mathbb{C}_{0}^{+}}$is in the point spectrum of $A_{Q}$; i.e., $A_{Q} x=\lambda x$ for some nonzero $x \in D(A)$. Then from (4.3) we obtain

$$
\begin{aligned}
2 \operatorname{Re} \lambda\langle Q x, x\rangle & =\left\langle A_{Q} x, Q x\right\rangle+\left\langle Q x, A_{Q} x\right\rangle \\
& =-\left\|B^{*} Q x\right\|^{2}-\|C x\|^{2} .
\end{aligned}
$$

Since $Q \geq 0$ and $\operatorname{Re} \lambda \geq 0$ we must have $B^{*} Q x=0=C x$, which implies that $\lambda x=A_{Q} x=A x$. So $\lambda$ is in the point spectrum of $A$. Suppose now that $\mu \in \overline{\mathbb{C}_{0}^{+}}$is in the residual spectrum of $A_{Q}$. Then by (4.6) $\bar{\mu}$ is in $\operatorname{P\sigma }\left(A_{Q}^{*}\right)=\operatorname{P\sigma }\left(A_{P}^{*}\right)$ and so there exists a $y \in D\left(A^{*}\right)$ such that $A_{P}^{*} y=\bar{\mu} y$. Now (4.2) can be reformulated as

$$
A_{P} P z+P A_{P}^{*} z=-P C^{*} C P z-B B^{*} z
$$

and substituting $z=y$ and taking the inner product with $y$ gives

$$
\begin{aligned}
2 \operatorname{Re} \mu\langle P y, y\rangle & =\left\langle A_{P}^{*} y, P y\right\rangle+\left\langle P y, A_{P}^{*} y\right\rangle \\
& =-\left\|B^{*} y\right\|^{2}-\|C P y\|^{2} .
\end{aligned}
$$

Since $\operatorname{Re} \mu \geq 0$ and $P \geq 0$, we must have $C P y=0=B^{*} y$, which implies that $\bar{\mu} y=A_{P}^{*} y=A^{*} y$ and so $\mu \in \sigma(A)$. Suppose now that $\lambda \in \overline{\mathbb{C}_{0}^{+}}$is in the continuous spectrum of $A_{Q}$. Then there exists a sequence $x_{n} \in D(A)$ with $\left\|x_{n}\right\|=1$ and $\left\|A_{Q} x_{n}-\lambda x_{n}\right\| \rightarrow 0$ as $n \rightarrow \infty$. Substituting in (4.3) we obtain

$$
\begin{aligned}
& \left\langle A_{Q} x_{n}-\lambda x_{n}, Q x_{n}\right\rangle+\left\langle Q x_{n}, A_{Q} x_{n}-\lambda x_{n}\right\rangle \\
& =-\left\|B^{*} Q x_{n}\right\|^{2}-\left\|C x_{n}\right\|^{2}-2 \operatorname{Re} \lambda\left\langle Q x_{n}, x_{n}\right\rangle .
\end{aligned}
$$

Since $Q \geq 0$ and $\operatorname{Re} \lambda \geq 0$, we deduce that $\left\|B^{*} Q x_{n}\right\|^{2} \leq 2\|Q\|\left\|x_{n}\right\|\left\|A_{Q} x_{n}-\lambda x_{n}\right\| \rightarrow$ 0 as $n \rightarrow \infty$. Thus

$$
\left\|A x_{n}-\lambda x_{n}\right\| \leq\left\|A_{Q} x_{n}-\lambda x_{n}\right\|+\left\|B B^{*} Q x_{n}\right\| \rightarrow 0
$$


as $n \rightarrow \infty$. So $\lambda$ is in the approximate point spectrum of $A$. The above shows that $\rho\left(A_{Q}\right) \cap \overline{\mathbb{C}_{0}^{+}} \subset \rho(A) \cap \overline{\mathbb{C}_{0}^{+}}$. Since by part 1 we have $\rho\left(A_{Q}\right)=\rho\left(A_{P}\right)$ this proves the assertion.

In [6] it was discovered that two interesting Riccati equations play a role in the solution to the Nehari problem.

THEOREM 4.4. Let $\Sigma(A, B, C, 0)$ be input and output stable and let $L_{1}, L_{2}$ be arbitrary bounded nonnegative solutions to the Lyapunov equations (2.1), (2.2), respectively. Let $\sigma>r^{\frac{1}{2}}\left(L_{1} L_{2}\right)$ and define $N_{\sigma}:=\left(I-\sigma^{-2} L_{1} L_{2}\right)^{-1}$. Then

1. $W:=N_{\sigma} L_{1}$ is a bounded nonnegative solution of the following Riccati equation for $z \in D\left(A^{*}\right)$ :

$$
W A^{*} z+A W z-\sigma^{-2} W C^{*} C W z+N_{\sigma} B B^{*} N_{\sigma}^{*} z=0
$$

2. $X:=L_{2} N_{\sigma}$ is a bounded nonnegative solution of the following Riccati equation for $z \in D(A)$ :

$$
A^{*} X z+X A z-\sigma^{-2} X B B^{*} X z+N_{\sigma}^{*} C^{*} C N_{\sigma} z=0
$$

3. the closed-loop systems $\Sigma\left(A_{W},\left[N_{\sigma} B ; W C^{*}\right], C\right)$ and $\Sigma\left(A_{X}, B,\left[C N_{\sigma} ; B^{*} X\right]\right)$ are system-stable, where $A_{W}=A-\sigma^{-2} W C^{*} C$ and $A_{X}=A-\sigma^{-2} B B^{*} X$;

4. $\sigma\left(A_{X}\right) \cap \mathbb{C}_{0}^{+} \subset \sigma(A) \cap \mathbb{C}_{0}^{+}$and $\sigma\left(A_{X}\right) \cap i \mathbb{R} \subset \sigma(A) \cap i \mathbb{R}$ and the closed-loop generators are related by $A_{X}=N_{\sigma}^{-1} A_{W} N_{\sigma}$.

Proof. 1. and 2. The proofs of Lemmas 4.1.24 and 8.3.2 in [4] show that $L_{1} D\left(A^{*}\right) \subset D(A), L_{2} D(A) \subset D\left(A^{*}\right), N_{\sigma} D(A) \subset D(A)$, and $N_{\sigma}^{*} D\left(A^{*}\right) \subset D\left(A^{*}\right)$. Thus $W D\left(A^{*}\right) \subset D(A)$ and $X D(A) \subset D\left(A^{*}\right)$. That $W$ satisfies (4.8) and $X$ satisfies (4.9) can be readily verified algebraically.

3. The conclusions about the stability of the closed-loop systems then follow from Theorem 4.2, noting that $\Sigma\left(A, 1 / \sigma B, C N_{\sigma}\right)$ is input stable and output stabilizable (with $\left.F=-1 / \sigma B^{*} X\right)$ and that $\Sigma\left(A, N_{\sigma} B, 1 / \sigma C\right)$ is output stable and input stabilizable (with $L=-1 / \sigma W C^{*}$ ).

4. Theorem 4.3 shows that $\sigma\left(A_{X}\right) \cap \mathbb{C}_{0}^{+} \subset \sigma(A) \cap \mathbb{C}_{0}^{+}$and $\sigma\left(A_{X}\right) \cap i \mathbb{R} \subset \sigma(A) \cap i \mathbb{R}$. To relate $A_{X}$ and $A_{W}$ consider

$$
\begin{aligned}
A_{X} N_{\sigma}^{-1} & =A\left(I-\sigma^{-2} L_{1} L_{2}\right)-\sigma^{-2} B B^{*} L_{2} \\
& =A-\sigma^{-2}\left(A L_{1}+B B^{*}\right) L_{2} \\
& =A+\sigma^{-2} L_{1} A^{*} L_{2} \\
& =A-\sigma^{-2} L_{1}\left(L_{2} A+C^{*} C\right) \\
& =\left(I-\sigma^{-2} L_{1} L_{2}\right) A-\sigma^{-2} L_{1} C^{*} C \\
& =N_{\sigma}^{-1} A_{W} .
\end{aligned}
$$

5. The spectral factor. As in [4] and [6] we shall approach the solution of the Nehari problem for the input and output stable state linear system $\Sigma(A, B, C, 0)$ with transfer function $\mathbf{G}$ by solving the following J-spectral factorization problem: find $\mathbf{X}$ such that

$$
\mathbf{P}(i \omega)^{*} J_{\sigma} \mathbf{P}(i \omega)=\mathbf{X}(i \omega) J_{1} \mathbf{X}(i \omega)^{*} \text { for almost all } \omega \in \mathbb{R}
$$

where

$$
\mathbf{P}(s)=\left[\begin{array}{cc}
I_{Y} & \mathbf{G}(s) \\
0 & I_{U}
\end{array}\right] \text { and } J_{\sigma}=\left[\begin{array}{cc}
I_{Y} & 0 \\
0 & -\sigma^{2} I_{U}
\end{array}\right]
$$


Here we introduce the candidate solution and give some properties. Let $L_{1}$ and $L_{2}$ be arbitrary bounded nonnegative solutions of the controllability and observability Lyapunov equations, respectively. For $\sigma>r^{\frac{1}{2}}\left(L_{1} L_{2}\right)$ we define $N_{\sigma}=\left(I-\sigma^{-2} L_{1} L_{2}\right)^{-1}$ and we introduce the state linear system

$$
\Sigma\left(A, \sigma^{-2} N_{\sigma}\left(\begin{array}{cc}
L_{1} C^{*} & -\sigma B
\end{array}\right),\left[\begin{array}{c}
C \\
B^{*} L_{2}
\end{array}\right],\left[\begin{array}{cc}
I_{Y} & 0 \\
0 & \sigma I_{U}
\end{array}\right]\right) .
$$

We denote the characteristic function of the state linear system (5.2) by $\mathfrak{X}$ and its transfer function by $\mathbf{X}$ and we prove the following lemma.

LEMMA 5.1. Let

$$
\mathfrak{P}(s)=\left[\begin{array}{cc}
I_{Y} & \mathfrak{G}(s) \\
0 & I_{U}
\end{array}\right] .
$$

Then $\mathfrak{R}(s)=\mathfrak{P}(s)^{*} J_{\sigma} \mathfrak{P}(s)-\mathfrak{X}(s) J_{1} \mathfrak{X}(s)^{*}$ satisfies the following for $s \in \rho(A)$ :

$$
\begin{aligned}
\mathfrak{R}(s)_{11}= & -2 \sigma^{-2} \operatorname{Re} s C(s I-A)^{-1} N_{\sigma} L_{1}(s I-A)^{-*} C^{*}, \\
\mathfrak{R}(s)_{12}= & -2 \sigma^{-2} \operatorname{Re} s C(s I-A)^{-1} N_{\sigma} L_{1}(s I-A)^{-*} L_{2} B, \\
\mathfrak{R}(s)_{21}= & \mathfrak{R}(s)_{12}^{*}, \\
\mathfrak{R}(s)_{22}= & -2 \operatorname{Re} s B^{*}(s I-A)^{-*} L_{2}(s I-A)^{-1} B \\
& -2 \sigma^{-2} \operatorname{Re} s B^{*} L_{2}(s I-A)^{-1} N_{\sigma} L_{1}(s I-A)^{-*} L_{2} B .
\end{aligned}
$$

Proof. We only prove the formula for $\mathfrak{R}(s)_{11}$; the proof for the other components is similar. We have on some right half-plane

$$
\begin{aligned}
\mathfrak{R}(s)_{11}= & I-\mathfrak{X}_{11}(s) \mathfrak{X}_{11}(s)^{*}+\mathfrak{X}_{12}(s) \mathfrak{X}_{12}(s)^{*} \\
= & -\sigma^{-4} C(s I-A)^{-1} W C^{*} C W(s I-A)^{-*} C^{*} \\
& +\sigma^{-2} C(s I-A)^{-1} N_{\sigma} B B^{*} N_{\sigma}^{*}(s I-A)^{-*} C^{*} \\
& -\sigma^{-2} C(s I-A)^{-1} W C^{*}-\sigma^{-2} C W(s I-A)^{-*} C \\
= & \sigma^{-2} C(s I-A)^{-1}\left(-\sigma^{-2} W C^{*} C W+N_{\sigma} B B^{*} N_{\sigma}^{*}\right)(s I-A)^{-*} C^{*} \\
& -\sigma^{-2} C(s I-A)^{-1} W C^{*}-\sigma^{-2} C W(s I-A)^{-*} C .
\end{aligned}
$$

Using (4.8) we obtain

$$
\begin{aligned}
\mathfrak{R}(s)_{11}= & \sigma^{-2} C(s I-A)^{-1}\left(-W A^{*}-A W\right)(s I-A)^{-*} C^{*} \\
& -\sigma^{-2} C(s I-A)^{-1} W C^{*}-\sigma^{-2} C W(s I-A)^{-*} C \\
= & \sigma^{-2} C(s I-A)^{-1}\left(W(s I-A)^{*}+(s I-A) W-2 \operatorname{Re} s W\right)(s I-A)^{-*} C^{*} \\
& -\sigma^{-2} C(s I-A)^{-1} W C^{*}-\sigma^{-2} C W(s I-A)^{-*} C \\
= & -2 \sigma^{-2} \operatorname{Re} s C(s I-A)^{-1} N_{\sigma} L_{1}(s I-A)^{-*} C^{*} .
\end{aligned}
$$

It is clear from the above that if $\sigma(A) \cap i \mathbb{R}$ has measure zero and $L_{1}, L_{2}$ are an arbitrary pair of solutions to the Lyapunov equations, then $\mathfrak{X}$ is a solution to (5.1). If, in addition, (5.2) is input or output stable, then it follows from Lemma 3.5 that $\mathbf{X}$ is a solution to (5.1). The following example shows that in general $\mathbf{X}$ need not provide a spectral factor.

Example 5.2. We consider an example from Curtain and Sasane [8] (see also Sasane [28]). The transfer function $\mathbf{G}_{0}(s)=\frac{1}{\sqrt{s^{2}+1}}$ was shown to have a realization 
$\Sigma\left(A, B, B^{*}, 0\right)$ on the state space $\ell_{2}(\mathbb{Z})$, where $A \in \mathcal{L}\left(\ell_{2}(\mathbb{Z})\right)$ and $B \in \ell_{2}(\mathbb{Z})$ are given by

$$
\begin{aligned}
A_{i, i+1} & =-1 / 2, A_{i+1, i}=1 / 2, A_{i, j}=0 \text { otherwise } \\
B_{0} & =1, B_{i}=0 \text { otherwise }
\end{aligned}
$$

The spectrum of $A$ is purely continuous and equals $[-i, i]$. The closed-loop system $\Sigma\left(A-B B^{*}, B, B^{*}, 0\right)$ is system-stable with the transfer function $\mathbf{G}(s)=\frac{1}{1+\sqrt{s^{2}+1}}$. It is continuous on the imaginary axis and it satisfies

$$
\begin{gathered}
\mathbf{G}(i \omega)+\mathbf{G}(i \omega)^{*}=2 \mathbf{G}(i \omega)^{*} \mathbf{G}(i \omega) \text { for }|\omega|>1, \\
\mathbf{G}(i \omega)=\mathbf{G}(i \omega)^{*}=\frac{1}{1+\sqrt{1-\omega^{2}}} \text { for }|\omega|<1 .
\end{gathered}
$$

The Lyapunov equations have solutions $L_{1}=L_{2}=1 / 2 I$, but note that it is known from [28] that these are not the observability or controllability gramians. The advantage of using these solutions is that the calculations are simple. In this specific case the state linear system (5.2) is

$$
\Sigma\left(A-B B^{*}, \sigma^{-2} N_{\sigma}[B / 2-\sigma B],\left[\begin{array}{c}
B^{*} \\
B^{*} / 2
\end{array}\right],\left[\begin{array}{ll}
1 & 0 \\
0 & \sigma
\end{array}\right]\right) .
$$

An easy calculation shows that for $s \in \rho\left(A-B B^{*}\right)=\rho(A)=\mathbb{C}-[-1,1]$ we have

$$
\mathfrak{X}(s)=\left[\begin{array}{ll}
1 & 0 \\
0 & \sigma
\end{array}\right]+\alpha\left[\begin{array}{cc}
2 & -4 \sigma \\
1 & -2 \sigma
\end{array}\right] \mathfrak{G}(s),
$$

where $\alpha=\frac{1}{4 \sigma^{2}-1}$. This, together with the stability properties of the state linear systems and Lemma 3.5, shows that we have for almost all $\omega \in \mathbb{R}$

$$
\mathbf{X}(i \omega)=\left[\begin{array}{ll}
1 & 0 \\
0 & \sigma
\end{array}\right]+\alpha\left[\begin{array}{ll}
2 & -4 \sigma \\
1 & -2 \sigma
\end{array}\right] \mathbf{G}(i \omega)
$$

It is now easily shown using (5.3) that (5.1) holds for $|\omega|>1$ and using (5.4) that (5.1) does not hold for $|\omega|<1$.

If we choose the smallest bounded nonnegative solutions to the Lyapunov equations we obtain stronger properties of the candidate spectral factor.

Lemma 5.3. If $\Sigma(A, B, C, 0)$ is output and input-output stable and $L_{2}=L_{C}$, the observability gramian of the system $\Sigma(A, B, C, 0)$, then (5.2) is output stable.

Proof. This follows from Lemma 3.14.

Lemma 5.4. If $\Sigma(A, B, C, 0)$ is system-stable and either $\sigma(A) \cap i \mathbb{R}$ has measure zero or $U$ and $Y$ are finite-dimensional, and $L_{1}=L_{B}$ and $L_{2}=L_{C}$, where $L_{B}, L_{C}$ are the controllability and observability gramians of the system $\Sigma(A, B, C, 0)$, respectively, then $\mathbf{X}$ satisfies (5.1).

Proof. It follows from Lemma 5.1 that on some right half-plane

$$
\left\langle\mathfrak{R}(s)\left[\begin{array}{l}
y \\
u
\end{array}\right],\left[\begin{array}{l}
y \\
u
\end{array}\right]\right\rangle=-\frac{2}{\sigma^{2}} \operatorname{Re} s\left(\|\alpha(s) y+\beta(s) u\|^{2}+\sigma^{2}\|\gamma(s) u\|^{2}\right),
$$

where $\mathfrak{R}$ is as in Lemma 5.1 and

$$
\begin{array}{ll}
\alpha(s)=M_{\sigma} L_{B}^{1 / 2}(s I-A)^{-*} C^{*}, & \beta(s)=M_{\sigma} L_{B}^{1 / 2}(s I-A)^{-*} L_{C} B, \\
\gamma(s)=L_{C}^{1 / 2}(s I-A)^{-1} B, & M_{\sigma}=\left(I-\frac{1}{\sigma^{2}} L_{B}^{1 / 2} L_{C} L_{B}^{1 / 2}\right)^{-1 / 2} .
\end{array}
$$


From the stability properties we can replace $\alpha, \beta$, and $\gamma$ in (5.5) by their holomorphic extensions (Lemmas 3.4 and 3.14). Then as in Lemma 3.12, using the real-analyticity property, it follows that the resulting equalities hold on $\mathbb{C}_{0}^{+}$.

Using Lemma 3.13 and Corollary 3.16 we see that the right-hand side of (5.5) converges to zero as $\operatorname{Re} s \rightarrow 0$ (here $\alpha, \beta$, and $\gamma$ are replaced by their holomorphic extensions). From this we obtain the J-spectral factorization (5.1).

In the remainder of this section we collect some properties of the spectral factor described by (5.2) and of its inverse system

$$
\Sigma\left(A, \sigma^{-2}\left[\begin{array}{ll}
-L_{1} C^{*} & B
\end{array}\right],\left[\begin{array}{c}
C \\
\sigma^{-1} B^{*} L_{2}
\end{array}\right] N_{\sigma},\left[\begin{array}{cc}
I_{Y} & 0 \\
0 & \sigma^{-1} I_{U}
\end{array}\right]\right) .
$$

We denote the characteristic function of the state linear system (5.6) by $\mathfrak{V}$ and its transfer function by $\mathbf{V}$. It is the inverse of $\mathfrak{X}$ in the following sense.

Lemma 5.5. Assume that $\Sigma(A, B, C, 0)$ is input and output stable. Then for $s \in \rho(A)$ we have $\mathfrak{V}(s) \mathfrak{X}(s)=I=\mathfrak{X}(s) \mathfrak{V}(s)$.

Proof. This follows from a straightforward calculation.

Lemma 5.6. Assume that $\Sigma(A, B, C, 0)$ is system-stable and that $L_{1}=L_{B}$, the controllability gramian of the system $\Sigma(A, B, C, 0)$. Then (5.6) is input stable.

Proof. This follows from Corollary 3.15 .

Lemma 5.7. Assume that $\Sigma(A, B, C, 0)$ is system-stable and that $L_{1}=L_{B}$ and $L_{2}=L_{C}$, the controllability and observability gramians of the system $\Sigma(A, B, C, 0)$, respectively. Then $\mathbf{V}(s) \mathbf{X}(s)=I=\mathbf{X}(s) \mathbf{V}(s)$ for all $s \in \mathbb{C}_{0}^{+}$.

Proof. From Lemmas 3.4, 5.3, 5.5, and 5.6 we have $\mathbf{V}(s) \mathbf{X}(s)=I=\mathbf{X}(s) \mathbf{V}(s)$ for all $s \in \mathbb{C}_{0}^{+} \cap \rho(A)$. From Lemmas 5.3 and 5.6 both $\mathbf{X}$ and $\mathbf{V}$ are holomorphic on $\mathbb{C}_{0}^{+}$and so the equality extends to this domain.

Lemma 5.8. Assume that $\Sigma(A, B, C, 0)$ is system-stable and that $L_{1}=L_{B}$ and $L_{2}=L_{C}$, the controllability and observability gramians of the system $\Sigma(A, B, C, 0)$, respectively. If either $\sigma(A) \cap i \mathbb{R}$ has measure zero or $U$ and $Y$ are finite-dimensional, then $\mathbf{V}(i \omega) \mathbf{X}(i \omega)=I=\mathbf{X}(i \omega) \mathbf{V}(i \omega)$ for almost all $\omega \in \mathbb{R}$.

Proof. This follows from Lemmas 5.3, 5.6, 5.7, and 3.5.

The $(2,2)$ component of $\mathfrak{V}$ plays a special role in what follows, and we need the following extra properties.

Lemma 5.9. Assume that $\Sigma(A, B, C, 0)$ is input and output stable. Then the system $\Sigma\left(A, \sigma^{-2} B, \sigma^{-1} B^{*} X, \sigma^{-1} I\right)$ is input stable, and its characteristic function $\mathfrak{V}_{22}(s)$ is invertible for $s \in \rho(A) \cap \rho\left(A_{X}\right)$. Its inverse is the characteristic function of the system-stable state linear system

$$
\Sigma\left(A_{X}, B,-\sigma^{-1} B^{*} X, \sigma I\right) \text {, with } X=L_{2} N_{\sigma} .
$$

Moreover, the transfer function $\mathbf{V}_{22}(s)$ is invertible for $s \in \mathbb{C}_{0}^{+}$, and its inverse is the transfer function of (5.7).

Proof. The input stability follows from that of $\Sigma(A, B, C, 0)$. The invertibility of the characteristic function $\mathfrak{V}_{22}$ is a simple calculation, and the stability property of (5.7) follows from Theorem 4.4. The invertibility of the transfer function follows as in Lemma 5.7.

Lemma 5.10. Assume that $\Sigma(A, B, C, 0)$ is input and output stable and that either $\sigma(A) \cap i \mathbb{R}$ has measure zero or $U$ and $Y$ are finite-dimensional. Then the boundary function of $\mathbf{V}_{22}$ is almost everywhere invertible, and its inverse is the boundary function of the transfer function of (5.7). 
Proof. This follows from Lemmas 5.9 and 3.5.

Dual results for $\mathfrak{X}_{11}(s)$ can be proved similarly.

Corollary 5.11. Assume that $\Sigma(A, B, C, 0)$ is input and output stable. Then the system $\Sigma\left(A, \sigma^{-2} W C^{*}, C, I\right)$ is output stable, and its characteristic function $\mathfrak{X}_{11}(s)$ is invertible for $s \in \rho(A) \cap \rho\left(A_{W}\right)$. Its inverse is the characteristic function of the system-stable state linear system

$$
\Sigma\left(A_{W},-\sigma^{-2} W C^{*}, C, I\right), \text { with } W=N_{\sigma} L_{1} .
$$

Moreover, the transfer function $\mathbf{X}_{11}(s)$ is invertible for $s \in \mathbb{C}_{0}^{+}$, and its inverse is the transfer function of (5.8).

Corollary 5.12. Assume that $\Sigma(A, B, C, 0)$ is input and output stable and that either $\sigma(A) \cap i \mathbb{R}$ has measure zero or $U$ and $Y$ are finite-dimensional. Then the boundary function of $\mathbf{X}_{11}$ is almost everywhere invertible, and its inverse is the boundary function of the transfer function of (5.8).

6. The central solution. We introduce the following state linear system

$$
\Sigma\left(A_{W}^{*}, L_{2} B,-\sigma^{-2} C W, 0\right),
$$

where $W$ is as in Theorem 4.4. We denote its characteristic function by $\mathfrak{Z}$ and its transfer function by $\mathbf{Z}$. The candidate solution to the Nehari problem is given by $\mathbf{K}_{c}(-s)=\mathbf{Z}(s)$. The state linear system (6.1) has the following properties.

LEMma 6.1. If $\Sigma(A, B, C, 0)$ is input and output stable, then the following hold:

1. The state linear system (6.1) is output stable.

2. The characteristic functions of the state linear system (6.1) and of the state linear system $\Sigma\left(A_{X}^{*},-\sigma^{-2} X B, C L_{1}, 0\right)$ coincide.

3. The state linear system $\Sigma\left(A_{X}^{*},-\sigma^{-2} X B, C L_{1}, 0\right)$ is input stable.

4. For $s \in \rho\left(A^{*}\right) \cap \rho\left(A_{W}^{*}\right)$ we have $\mathfrak{Z}(s)=\mathfrak{V}_{21}(\bar{s})^{*} \mathfrak{V}_{22}^{-1}(\bar{s})^{*}$.

Proof. 1. This follows from Theorem 4.4, part 3.

2. This is an easy calculation using Theorem 4.4, part 4 .

3. This follows from Theorem 4.4, part 3.

4. This is a simple calculation.

The above shows that we have one realization of $\mathbf{Z}$ that is output stable and another that is input stable. We now show that $\mathbf{Z}$ is in $\mathbf{H}_{\infty}$ under the assumption A1.

TheOREm 6.2. If $\Sigma(A, B, C, 0)$ is system-stable and $\sigma(A) \cap i \mathbb{R}$ has measure zero, then $\mathbf{Z} \in \mathbf{H}_{\infty}(\mathcal{L}(U, Y))$ and with $\mathbf{K}_{c}(-s)=\mathbf{Z}(s)$ we have

$$
\left\|\mathbf{G}+\mathbf{K}_{c}\right\|_{\infty} \leq \sigma .
$$

Proof. It follows from Lemma 6.1, part 4, and Theorem 4.4, part 4, that for almost all $\omega \in \mathbb{R}$ we have

$$
\mathfrak{K}_{c}(i \omega)=\mathfrak{V}_{21}(i \omega)^{*} \mathfrak{V}_{22}(i \omega)^{-*} .
$$

From Lemma 5.1 we see that for almost all $\omega \in \mathbb{R}$ we have

$$
\mathfrak{P}(i \omega)^{*} J_{\sigma} \mathfrak{P}(i \omega)=\mathfrak{X}(i \omega) J_{1} \mathfrak{X}(i \omega)^{*} .
$$

From (6.2) we obtain for almost all $\omega \in \mathbb{R}$

$$
\left[\begin{array}{c}
\mathfrak{G}(i \omega)+\mathfrak{K}_{c}(i \omega) \\
I
\end{array}\right]=\mathfrak{P}(i \omega) \mathfrak{V}(i \omega)^{*}\left[\begin{array}{c}
0 \\
\mathfrak{V}_{22}(i \omega)^{-*}
\end{array}\right]
$$


So

$$
\begin{aligned}
& \left(\mathfrak{G}(i \omega)+\mathfrak{K}_{c}(i \omega)\right)^{*}\left(\mathfrak{G}(\mathfrak{i} \omega)+\mathfrak{K}_{\mathfrak{c}}(\mathfrak{i} \omega)\right)-\sigma^{2} I \\
& =\left[\begin{array}{c}
\mathfrak{G}(\mathfrak{i} \omega)+\mathfrak{K}_{\mathfrak{c}}(\mathfrak{i} \omega) \\
I
\end{array}\right]^{*} J_{\sigma}\left[\begin{array}{c}
\mathfrak{G}(\mathfrak{i} \omega)+\mathfrak{K}_{\mathfrak{c}}(\mathfrak{i} \omega) \\
I
\end{array}\right] \\
& =\left[\begin{array}{c}
0 \\
\mathfrak{V}_{22}(i \omega)^{-*}
\end{array}\right]^{*} \mathfrak{V}(i \omega) \mathfrak{P}(i \omega)^{*} J_{\sigma} \mathfrak{P}(i \omega) \mathfrak{V}(i \omega)^{*}\left[\begin{array}{c}
0 \\
\mathfrak{V}_{22}(i \omega)^{-*}
\end{array}\right] \\
& =\left[\begin{array}{c}
0 \\
\mathfrak{V}_{22}(i \omega)^{-*}
\end{array}\right]^{*}\left[\begin{array}{cc}
I & 0 \\
0 & -I
\end{array}\right]\left[\begin{array}{c}
0 \\
\mathfrak{V}_{22}(i \omega)^{-*}
\end{array}\right],
\end{aligned}
$$

where in the last step we have used the spectral factorization (6.3). The above shows that

$$
\left\|\mathfrak{G}(i \omega)+\mathfrak{K}_{c}(i \omega)\right\|^{2}-\sigma^{2}=-\left\|\mathfrak{V}_{22}(i \omega)^{-*}\right\|^{2},
$$

and so for almost all $\omega \in \mathbb{R}$

$$
\left\|\mathfrak{G}(i \omega)+\mathfrak{K}_{c}(i \omega)\right\| \leq \sigma,
$$

which implies that $\mathfrak{G}+\mathfrak{K}_{c} \in \mathbf{L}_{\infty}(i \mathbb{R} ; \mathcal{L}(U, Y))$. Since $\mathbf{G} \in \mathbf{H}_{\infty}(\mathcal{L}(U, Y))$ and by Lemma $3.5 \mathfrak{G}$ coincides almost everywhere with the boundary function of $\mathbf{G}$ on the imaginary axis, we have $\mathfrak{G} \in \mathbf{L}_{\infty}(i \mathbb{R} ; \mathcal{L}(U, Y))$ and thus $\mathfrak{K}_{c} \in \mathbf{L}_{\infty}(i \mathbb{R} ; \mathcal{L}(U, Y))$. From this it follows that $\mathfrak{Z} \in \mathbf{L}_{\infty}(i \mathbb{R} ; \mathcal{L}(U, Y))$. This, together with the output stability of the state linear system (6.1) from Lemma 6.1 using Lemmas 3.5 and 3.6, shows that $\mathbf{Z} \in \mathbf{H}_{\infty}(\mathcal{L}(U, Y))$. Thus with $\mathbf{K}_{c}(-s)=\mathbf{Z}(s)$ we obtain $\left\|\mathbf{G}+\mathbf{K}_{c}\right\|_{\infty} \leq \sigma$.

Our main result in this section is to show that under assumption A2, $\mathbf{Z} \in \mathbf{H}_{\infty}$ and $\mathbf{K}_{c}$ solves the suboptimal Nehari problem.

TheOrem 6.3. Assume that $\Sigma(A, B, C, 0)$ is system-stable and that $L_{1}=L_{B}$ and $L_{2}=L_{C}$, the controllability and observability gramians of the system $\Sigma(A, B, C, 0)$, respectively, and that $U$ and $Y$ are finite-dimensional. Then $\mathbf{Z} \in \mathbf{H}_{\infty}(\mathcal{L}(U, Y))$ and with $\mathbf{K}_{c}(-s)=\mathbf{Z}(s)$ we have

$$
\left\|\mathbf{G}+\mathbf{K}_{c}\right\|_{\infty} \leq \sigma .
$$

Proof. The idea is to follow the lines of the proof of Theorem 6.2, replacing the characteristic functions by their corresponding transfer functions. So all we need to show is that the following two key properties hold for almost all $\omega \in \mathbb{R}$ :

$$
\begin{gathered}
\mathbf{Z}(i \omega)=\mathbf{V}_{21}(-i \omega)^{*} \mathbf{V}_{22}^{-1}(-i \omega)^{*} \\
\mathbf{P}(i \omega)^{*} J_{\sigma} \mathbf{P}(i \omega)=\mathbf{X}(i \omega) J_{1} \mathbf{X}(i \omega)^{*}
\end{gathered}
$$

Since (6.6) has already been shown in Lemma 5.4, it remains to show only (6.5). By Lemma 6.1, part 4, on some right half-plane we have $\mathbf{Z}(s)=\mathbf{V}_{21}(\bar{s})^{*} \mathbf{V}_{22}^{-1}(\bar{s})^{*}$. Using Lemma 6.1, part 1, Lemma 5.6, and Lemma 5.9 this equality holds on $\mathbb{C}_{0}^{+}$(all functions are holomorphic on $\mathbb{C}_{0}^{+}$). Lemmas 3.5 and 5.10 now give (6.5).

Under assumption A2 we can show that $\mathbf{Z}$ has a realization as a system-stable state linear system.

Corollary 6.4. Assume that $\Sigma(A, B, C, 0)$ is system-stable and that $L_{1}=L_{B}$ and $L_{2}=L_{C}$, the controllability and observability gramians of the system $\Sigma(A, B, C, 0)$, 
respectively, and that $U$ and $Y$ are finite-dimensional. Then $\Sigma\left(A_{W}^{*}, L_{2} B,-\sigma^{-2} C W, 0\right)$ and $\Sigma\left(A_{X}^{*},-\sigma^{-2} X B, C L_{1}, 0\right)$ are system-stable state linear systems.

Proof. We have already shown in Lemma 6.1 and Theorem 6.3 that $\Sigma\left(A_{W}^{*}, L_{C} B\right.$, $\left.-\sigma^{-2} C W, 0\right)$ is output and input-output stable. The input stability follows from the identity

$$
B^{*} L_{C}\left(s I-A_{W}\right)^{-1}=B^{*} L_{C}(s I-A)^{-1}-\sigma^{-2} B^{*} L_{C}\left(s I-A_{W}\right)^{-1} W C^{*} C(s I-A)^{-1}
$$

and the fact $B^{*} L_{C}(s I-A)^{-1} z \in \mathbf{H}_{2}(Y)$ (Lemma 3.14), that $B^{*} L_{C}\left(s I-A_{W}\right)^{-1} W C^{*} \in$ $\mathbf{H}_{\infty}(\mathcal{L}(Y, U))$ (the input-output stability shown earlier), and that $C(s I-A)^{-1} z \in$ $\mathbf{H}_{2}(Y)$ for all $z \in Z$. The proof for the other realization is similar.

7. Parametrization of solutions. First we give a parameterization of a family of solutions to the Nehari problem in terms of the transfer function of (5.6) and an $\mathbf{H}_{\infty}$ parameter under assumption $\mathrm{A} 2$.

TheOREM 7.1. Let $\Sigma(A, B, C, 0)$ be system-stable with transfer function $\mathbf{G}$ and let $L_{B}$ and $L_{C}$ be the controllability and observability gramians, respectively. Assume that $U$ and $Y$ are finite-dimensional. For $\sigma>r^{\frac{1}{2}}\left(L_{B} L_{C}\right)$ define

$$
\left[\begin{array}{l}
\mathbf{R}_{1}(s) \\
\mathbf{R}_{2}(s)
\end{array}\right]=\mathbf{V}(\bar{s})^{*}\left[\begin{array}{c}
\mathbf{Q}(-s) \\
I_{U}
\end{array}\right]
$$

where $\mathbf{Q}(-s) \in \mathbf{H}_{\infty}(\mathcal{L}(U, Y))$. If $\|\mathbf{Q}\|_{\infty} \leq 1$, then $\mathbf{K}(-s)=\mathbf{R}_{1}(s) \mathbf{R}_{2}(s)^{-1} \in$ $\mathbf{H}_{\infty}(\mathcal{L}(U, Y))$ and satisfies

$$
\|\mathbf{K}+\mathbf{G}\|_{\infty} \leq \sigma
$$

We prove this in a series of lemmas.

LEMma 7.2. Under the assumptions of Theorem 7.1 we have the following. For all $s \in \mathbb{C}_{0}^{+}$and almost all $s \in i \mathbb{R}$ we have $\left\|\mathbf{V}_{12}(s) \mathbf{V}_{22}^{-1}(s)\right\|<1$.

Proof. Consider the state linear system $\Sigma\left(A, \sigma^{-1} B, C N_{\sigma}, 0\right)$, and denote its transfer function by $\mathbf{E}$. The control Riccati equation (4.1) corresponding to this system is precisely (4.9). From the proof of Theorem 4.2, specifically by (4.5), we have $\|[\mathbf{N} ; \mathbf{M}]\|_{\infty} \leq 1$, where $[\mathbf{N} ; \mathbf{M}]$ is the transfer function of the closed-loop system $\Sigma\left(A_{X}, \sigma^{-1} B,\left[C N_{\sigma},-\sigma^{-1} B^{*} X\right],[0 ; I]\right)$. It is easily calculated that $\mathbf{V}_{12}(s) \mathbf{V}_{22}^{-1}(s)=$ $\mathbf{N}(s)$. It also easily seen that $\mathbf{N}(s)=\mathbf{E}(s) \mathbf{M}(s)$ on some right half-plane. By stability this extends to the right half-plane and by taking nontangential limits to almost everywhere on the imaginary axis.

From the above inequality we obtain for almost all $\omega \in \mathbb{R}$ that $\|\mathbf{N}(i \omega)\| \leq 1$. We show that, in fact, strict inequality holds. Suppose, on the contrary, that $\left\|\mathbf{N}\left(i \omega_{0}\right)\right\|=$ 1. Then there would exist a sequence $u_{n}$ with norm one such that $\left\|\mathbf{N}\left(i \omega_{0}\right) u_{n}\right\| \rightarrow 1$. From the above $\mathbf{H}_{\infty}$ bound we conclude that $\left\|\mathbf{M}\left(i \omega_{0}\right) u_{n}\right\| \rightarrow 0$. Since for almost all $\omega \in \mathbb{R}$ we have $\mathbf{N}(i \omega)=\mathbf{E}(i \omega) \mathbf{M}(i \omega)$, we obtain $\left\|\mathbf{N}\left(i \omega_{0}\right) u_{n}\right\| \rightarrow 0$. This gives the desired contradiction.

We now extend this inequality to $s \in \mathbb{C}_{0}^{+}$. From the above we know that $\left\|\mathbf{V}_{12} \mathbf{V}_{22}^{-1}\right\|_{\infty}=\|\mathbf{N}\|_{\infty} \leq 1$. Suppose that there exists a point $s_{0} \in \mathbb{C}_{0}^{+}$such that $\left\|\mathbf{V}_{12}\left(s_{0}\right) \mathbf{V}_{22}^{-1}\left(s_{0}\right)\right\|=1$. Then $\left\|\mathbf{V}_{12}(s) \mathbf{V}_{22}^{-1}(s)\right\|$ has a maximum in $\mathbb{C}_{0}^{+}$and is therefore constant by the maximum modulus principle; see, e.g., [17, Theorem 3.13.1, p. 100]. This implies that $\left\|\mathbf{V}_{12}(s) \mathbf{V}_{22}^{-1}(s)\right\|=1$ for all $s \in \mathbb{C}_{0}^{+}$. But then the boundary function would have norm one almost everywhere, and we have shown that this is not true. 
The next lemma ensures that $\mathbf{K}$ is well defined.

Lemma 7.3. Under the assumptions of Theorem $7.1, \mathbf{R}_{2}(s)$ is invertible for all $s \in \mathbb{C}_{0}^{+}$and almost all $s \in i \mathbb{R}$.

Proof. Define $\mathbf{P}(s):=\mathbf{Q}(-\bar{s})^{*}$. Then $\mathbf{P} \in \mathbf{H}_{\infty}(\mathcal{L}(U, Y))$, and $\|\mathbf{P}\|_{\infty} \leq 1$. Next, using Lemma 7.2 we obtain $\left\|\mathbf{P}(s) \mathbf{V}_{12}(s) \mathbf{V}_{22}^{-1}(s)\right\|<1$. From this we see that $(I+$ $\left.\mathbf{P}(s) \mathbf{V}_{12}(s) \mathbf{V}_{22}^{-1}(s)\right)^{-1}=\mathbf{V}_{22}(s)\left(\mathbf{V}_{22}(s)+\mathbf{P}(s) \mathbf{V}_{12}(s)\right)^{-1}$ exists. Hence $\mathbf{T}:=\left(\mathbf{V}_{22}+\right.$ $\left.\mathbf{P} \mathbf{V}_{12}\right)^{-1}$ exists. Since we have $\mathbf{R}_{2}(s)^{-1}=\mathbf{T}(\bar{s})^{*}$ we have that $\mathbf{R}_{2}(s)^{-1}$ exists.

Next we prove Theorem 7.1 under the assumption $\|\mathbf{Q}\|_{\infty}<1$.

Lemma 7.4. Theorem 7.1 is true for all $\mathbf{Q}(-s) \in \mathbf{H}_{\infty}(\mathcal{L}(U, Y))$ with $\|\mathbf{Q}\|_{\infty}<1$.

Proof. We show that $\mathbf{R}_{2}^{-1} \in \mathbf{H}_{\infty}(\mathcal{L}(U))$. This follows as the proof of Lemma 7.3 using that $\mathbf{H}_{\infty}$ is a Banach algebra: from $\left\|\mathbf{P V}_{12} \mathbf{V}_{22}^{-1}\right\|_{\infty}<1$ we conclude that $\left(I+\mathbf{P} \mathbf{V}_{12} \mathbf{V}_{22}^{-1}\right)^{-1} \in \mathbf{H}_{\infty}$, and using that $\mathbf{V}_{22}^{-1} \in \mathbf{H}_{\infty}$ by Lemma 5.9 it follows that $\mathbf{R}_{2}^{-1} \in \mathbf{H}_{\infty}(\mathcal{L}(U))$.

$\mathbf{K}(-s):=\mathbf{R}_{1}(s) \mathbf{R}_{2}(s)^{-1}$ defines an $\mathbf{L}_{\infty}$ function which satisfies $\|\mathbf{G}+\mathbf{K}\|_{\infty} \leq \sigma$. The proof is similar to that of Theorem 6.2.

Last we show that $\mathbf{K}(-s) \in \mathbf{H}_{\infty}(\mathcal{L}(U, Y))$.

Since for all $s \in \mathbb{C}_{0}^{+}$we have $\mathbf{V}(s) \mathbf{X}(s)=I$, we obtain

$$
\mathbf{X}_{11}(s) \mathbf{V}_{11}(s)+\mathbf{X}_{12}(s) \mathbf{V}_{21}(s)=I
$$

and

$$
\mathbf{X}_{11}(s) \mathbf{V}_{12}(s)+\mathbf{X}_{12}(s) \mathbf{V}_{22}(s)=0
$$

from which we obtain

$$
\mathbf{V}_{11}(s)=\mathbf{X}_{11}(s)^{-1}+\mathbf{V}_{12}(s) \mathbf{V}_{22}(s)^{-1} \mathbf{V}_{21}(s) .
$$

So

$$
\begin{aligned}
\mathbf{R}_{1}(s) & =\mathbf{V}_{21}(\bar{s})^{*}+\mathbf{V}_{11}(\bar{s})^{*} \mathbf{Q}(-s) \\
& =\mathbf{X}_{11}(\bar{s})^{-*} \mathbf{Q}(-s)+\mathbf{V}_{21}(\bar{s})^{*}\left(I+\mathbf{V}_{22}(\bar{s})^{-*} \mathbf{V}_{12}(\bar{s})^{*} \mathbf{Q}(-s)\right) \\
& =\mathbf{X}_{11}(\bar{s})^{-*} \mathbf{Q}(-s)+\mathbf{V}_{21}(\bar{s})^{*} \mathbf{V}_{22}(\bar{s})^{-*} \mathbf{R}_{2}(s) \\
& =\mathbf{X}_{11}(\bar{s})^{-*} \mathbf{Q}(-s)+\mathbf{K}_{c}(-s) \mathbf{R}_{2}(s)
\end{aligned}
$$

and so

$$
\mathbf{K}(-s)=\mathbf{X}_{11}(\bar{s})^{-*} \mathbf{Q}(-s) \mathbf{R}_{2}(s)^{-1}+\mathbf{K}_{c}(-s) .
$$

Now $\mathbf{K}_{c}(-s) \in \mathbf{H}_{\infty}$ by Theorem $6.3, \mathbf{X}_{11}^{-1} \in \mathbf{H}_{\infty}$ by Corollary $5.11, \mathbf{R}_{2}(s)^{-1} \in \mathbf{H}_{\infty}$ as we proved above, and $\mathbf{Q}(-s) \in \mathbf{H}_{\infty}$ is given. So $\mathbf{K}(-s) \in \mathbf{H}_{\infty}(U, Y)$.

The proof for the case $\|\mathbf{Q}\|_{\infty} \leq 1$ follows the approach in [1].

Lemma 7.5. Theorem 7.1 is true for all $\mathbf{Q}(-s) \in \mathbf{H}_{\infty}(\mathcal{L}(U, Y))$ with $\|\mathbf{Q}\|_{\infty} \leq 1$.

Proof. We first note that $\mathbf{H}_{\infty}$ is weak-* closed in $\mathbf{L}_{\infty}$. In the case of the unit disc and the unit circle instead of the right half-plane and the imaginary axis, this follows as in [27, p. 197]. The case we consider follows, using a Möbius transform.

Next we note that if a bounded sequence $F_{n}$ in $\mathbf{L}_{\infty}$ converges pointwise to $F \in$ $\mathbf{L}_{\infty}$, then $F_{n}$ converges to $F$ in the weak-* topology. In the case of the unit disc and the unit circle instead of the right half-plane and the imaginary axis, this follows as in [1, Proposition 2.3]. The case we consider follows, using a Möbius transformation. 
Using these two results, we prove the lemma. For $t \in(0,1)$ define $\mathbf{Q}_{t}:=t \mathbf{Q}$. Then $\left\|\mathbf{Q}_{t}\right\| \leq t<1$. Define $\mathbf{K}_{t}$ in terms of $\mathbf{Q}_{t}$. Then by Lemma 7.4 we have $\mathbf{K}_{t} \in \mathbf{H}_{\infty}$. If $t \rightarrow 1$, then for almost all $\omega \in \mathbb{R}$ we have $\mathbf{K}_{t}(i \omega) \rightarrow \mathbf{K}(i \omega)$. Since $\mathbf{K}_{t}$ is bounded in norm by $\|\mathbf{G}\|_{\infty}+\sigma$, the limit function (which is well defined by Corollary 7.3) is in $\mathbf{L}_{\infty}$. By the above this implies that $\mathbf{K}_{t}$ converges to $\mathbf{K}$ in the weak-* topology. Since $\mathbf{H}_{\infty}$ is closed in the weak-* topology and $\mathbf{K}_{t} \in \mathbf{H}_{\infty}$, we obtain $\mathbf{K} \in \mathbf{H}_{\infty}$.

Remark 7.6. It is clear from the results in the previous section and from the proof of Theorem 7.1 that the conclusions also hold under assumption A1: $\sigma(A) \cap i \mathbb{R}$ has measure zero. In this case $L_{B}$ and $L_{C}$ can be replaced by arbitrary bounded nonnegative solutions of the Lyapunov equations, and the assumption that $U$ and $Y$ should be finite-dimensional is redundant.

8. Well-posed linear systems and reciprocals. In this section we solve the suboptimal Nehari problem via the reciprocal system as in Curtain and Sasane [9].

First we briefly review the definitions of a well-posed linear system (see Weiss [32], Staffans [29]). Given an $\mathcal{L}(U, Y)$-valued function $\mathbf{G}$ that is holomorphic and uniformly bounded on some right half-plane, there exist operators $A, B, C$ such that

- $A$ is the infinitesimal generator of a strongly continuous semigroup $T(\cdot)$ on a separable Hilbert space $Z$;

- $C \in \mathcal{L}(D(A), Y)$ is an admissible observation operator with respect to $T(\cdot)$; i.e., given $\tau>0$, there exists a $\gamma>0$ such that

$$
\int_{0}^{\tau}\|C T(t) z\|^{2} \mathrm{~d} t \leq \gamma\|z\|^{2} \quad \forall z \in D(A) ;
$$

- $B^{*} \in \mathcal{L}\left(D\left(A^{*}\right), U\right)$, and $B$ is an admissible control operator for $T(\cdot)$; i.e., for any $\tau>0$, there exists a $\beta>0$ such that for all $u \in \mathbf{L}_{2}(0, \tau ; U)$

$$
\left\|\int_{0}^{\tau} T(t-s) B u(s) \mathrm{d} s\right\|^{2} \leq \beta \int_{0}^{\tau}\|u(t)\|^{2} \mathrm{~d} t ;
$$

- The operators $A, B, C$ should be such that

$$
\mathbf{G}(s)-\mathbf{G}(\alpha)=(\alpha-s) C(s I-A)^{-1}(\alpha I-A)^{-1} B
$$

for any $\alpha, s$ larger than the growth bound of the semigroup $T$.

A triple $A, B, C$ that satisfies the above conditions is called a realization of the function G. Such a realization is not unique. A well-posed linear system is specified by operators $A, B, C$ and a transfer function $\mathbf{G}$ that satisfy the above conditions.

The expression (8.1) is defined for all $s \in \rho(A)$, and as in section 2 to avoid confusion, we call this the characteristic function and denote it by $\mathfrak{G}$. If the admissibility definitions can be extended to $\tau=\infty$, then the term infinite-time admissible is used. Well-posed linear systems form a nice generalization of state linear systems, and the concepts of infinite-time admissibility are the natural extensions of input and output stability in Definition 2.3, and we shall use the terms input and output stability. In Grabowski [15] and Hansen and Weiss [16] it is shown that Lemma 2.6 generalizes perfectly to well-posed linear systems and in Curtain [11] that Lemma 3.4 also applies to well-posed linear systems. We call $\Sigma$ a system-stable well-posed linear system if it is input stable and output stable and $\mathbf{G} \in \mathbf{H}_{\infty}(\mathcal{L}(U, Y))$.

The concept of a reciprocal system was introduced in [10].

DEFINITION 8.1. Suppose that the well-posed linear system $\Sigma$ with generating operators $A, B, C$ and transfer function $\mathbf{G}$ is such that $0 \in \rho(A)$. Its reciprocal system is the state linear system $\Sigma\left(A^{-1}, A^{-1} B,-C A^{-1}, \mathfrak{G}(0)\right)$. 
The justification for this definition is the nice relationship between the well-posed linear system and its reciprocal system shown in [11, Lemma 3.2].

THEOREM 8.2. Suppose that $A, B, C$ are the generating operators of a well-posed linear system $\Sigma$ with transfer function $\mathbf{G}$ and zero is in the resolvent set of $A$. Denote the characteristic function of its reciprocal system by $\mathfrak{G}_{r}$ and the transfer function of its reciprocal system by $\mathbf{G}_{r}$. Then the following hold:

1. $\mathfrak{G}(s)=\mathfrak{G}_{r}\left(\frac{1}{s}\right)$ whenever $s$ is in the resolvent set of $A$.

2. $\Sigma$ is output stable if and only if its reciprocal system is output stable. If they are output stable, then their observability gramians are identical.

3. $\Sigma$ is input stable if and only if its reciprocal system is input stable. If they are input stable, then their controllability gramians are identical.

4. The well-posed linear system is system-stable if and only if its reciprocal system is system-stable. In this case, we have $\mathbf{G}(s)=\mathbf{G}_{r}\left(\frac{1}{s}\right)$ for $s \in \mathbb{C}_{0}^{+}$.

The advantages of working with the reciprocal system are that all its generating operators are bounded and the close connections with the original well-posed linear system give us the following result.

THEOREM 8.3. Let $\Sigma$ be a system-stable well-posed linear system with generating operators $A, B, C$ and transfer function $\mathbf{G}$ and assume that $0 \in \rho(A)$. Let $\mathbf{G}_{r}$ denote the transfer function of its reciprocal system $\Sigma\left(A^{-1}, A^{-1} B,-C A^{-1}, \mathfrak{G}(0)\right)$. Then $\mathbf{K}(-s) \in \mathbf{H}_{\infty}(\mathcal{L}(U, Y))$ satisfies

$$
\|\mathbf{G}+\mathbf{K}\| \leq \sigma
$$

if and only if $\mathbf{K}_{r}(-s) \in \mathbf{H}_{\infty}(\mathcal{L}(U, Y))$ satisfies

$$
\left\|\mathbf{G}_{r}-\mathfrak{G}(0)+\mathbf{K}_{r}\right\| \leq \sigma
$$

where $\mathbf{K}(s)=\mathbf{K}_{r}\left(\frac{1}{s}\right)-\mathfrak{G}(0)$ for all $s \in \mathbb{C}_{0}^{+}$and almost all $s \in i \mathbb{R}$.

Proof. From Theorem 8.2, part 4, we have $\mathbf{G}(s)=\mathbf{G}_{-}\left(\frac{1}{s}\right)$ for $s \in \mathbb{C}_{0}^{+}$and by input-output stability this extends to almost everywhere on $i \mathbb{R}$. So for all $s \in \mathbb{C}_{0}^{+}$and almost all $s \in i \mathbb{R}$ we have

$$
\mathbf{K}(-s)+\mathbf{G}(s)=\mathbf{K}_{r}\left(-\frac{1}{s}\right)+\left[\mathbf{G}_{r}\left(\frac{1}{s}\right)-\mathfrak{G}(0)\right] .
$$

Thus

$$
\begin{aligned}
\sup _{\mathbb{C}_{0}^{+}}\|\mathbf{K}(-s)+\mathbf{G}(s)\| & =\sup _{\mathbb{C}_{0}^{+}}\left\|\mathbf{K}_{r}\left(-\frac{1}{s}\right)+\mathbf{G}_{r}\left(\frac{1}{s}\right)-\mathfrak{G}(0)\right\| \\
& =\sup _{\mathbb{C}_{0}^{+}}\left\|\mathbf{K}_{r}(-s)+\mathbf{G}_{r}(s)-\mathfrak{G}(0)\right\|,
\end{aligned}
$$

which proves the claim.

Remark 8.4. 1. Since $\mathbf{G}_{r}\left(\frac{1}{s}\right)-\mathfrak{G}(0)$ is the transfer function of the system-stable state linear system $\Sigma\left(A^{-1}, A^{-1} B,-C A^{-1}, 0\right)$, the results on state linear systems in this article generalize to well-posed linear systems in an obvious way. Note that the formulas that we so obtain are not the same as for the case of state linear systems but are in terms of the generating operators of the reciprocal system. The analogous formulas for the well-posed linear system need not be well defined.

2. Finally, we remark that the assumption in Theorem 8.3 that $0 \in \rho(A)$ can be relaxed. The arguments in this section can be adapted to the alternative assumption 
that $i \omega \in \rho(A)$ for some real $\omega$. Denoting $A_{\omega}=A-i \omega I$, we introduce the $\omega$ reciprocal systems $\Sigma\left(A_{\omega}^{-1}, A_{\omega}^{-1},-C A_{\omega}^{-1}, \mathbf{G}(i \omega)\right)$ with transfer function $\mathbf{G}_{r}^{\omega}$. Noting that $\mathbf{G}(s+i \omega)=\mathbf{G}_{r}^{\omega}\left(\frac{1}{s}\right)$, we can obtain connections between the Nehari problem for $\Sigma$ and this new reciprocal system. By proving our results on state linear systems in discrete time and using the Cayley transform, one can even obtain Theorem 8.3 without any assumption on the spectrum.

9. Appendix. In this appendix we study real-analytic functions on a complex Banach space $E$ following Dieudonné [12]. A function $f: \Omega \subset \mathbb{R}^{2} \rightarrow E$ with $\Omega$ open is called real-analytic if at every point $\omega \in \Omega$ there exist vectors $c_{i, j} \in E$ such that

$$
f(x, y)=\sum_{i, j \in \mathbb{N}^{2}} c_{i, j}\left(x-\omega_{1}\right)^{i}\left(y-\omega_{2}\right)^{j}
$$

for all points $(x, y)$ in a neighborhood of $\omega$, the series converging absolutely in this neighborhood.

Consider a holomorphic function $h: \Omega \subset \mathbb{C} \rightarrow E$. It follows from Goursat's theorem [12, section 9.10, Problem 1] that at every point $h$ can be expanded in an absolutely convergent power series in the complex variable $z$. Define $h_{\mathbb{R}}: \Omega \subset \mathbb{R}^{2} \rightarrow E$ by $h_{\mathbb{R}}(x, y):=h(x+i y)$. It is easily seen that $h_{\mathbb{R}}$ is real-analytic: the series expansion in $x$ and $y$ follows from the series expansion in $x+i y$. We further note that if $g: \Omega \rightarrow \mathbb{C}$ is real-analytic, then so is $\bar{g}$.

COROLLARY 9.1. Using the notation and assumptions of Lemma 3.12, we have that $(x, y) \mapsto\left\langle\widehat{\mathcal{B}}(x+i y) u, L_{C} B u\right\rangle$ and $(x, y) \mapsto\left\langle L_{C} B u, \widehat{\mathcal{B}}(x+i y) u\right\rangle$ are real-analytic on the right half-plane.

Proof. With $h(s)=g(s)=\left\langle\widehat{\mathcal{B}}(s) u, L_{C} B u\right\rangle$ this follows from the above discussion.

We have the following characterization of real-analyticity.

LEMMA 9.2. $f: \Omega \subset \mathbb{R}^{2} \rightarrow E$ is real-analytic if and only if there exists an open set $\Omega_{\mathbb{C}} \subset \mathbb{C}^{2}$ such that $\Omega_{\mathbb{C}} \cap \mathbb{R}^{2}=\Omega$ and a holomorphic function $f_{\mathbb{C}}: \Omega_{\mathbb{C}} \rightarrow$ E such that $\left.f_{\mathbb{C}}\right|_{\Omega}=f$.

Proof. This follows from [12, subsection 9.4.5, p. 209] and Goursat's theorem [12, section 9.10, Problem 1].

TheOREM 9.3. If $f, g: \Omega \rightarrow H$ are real-analytic, then $\langle f, g\rangle$ is real-analytic.

Proof. Since $f$ is real-analytic, there exists a holomorphic function $f_{\mathbb{C}}$ of which $f$ is the restriction. Since $\bar{g}$ is real-analytic, there exists a holomorphic function $\bar{g}_{\mathbb{C}}$ of which $\bar{g}$ is the restriction. We thus have that $\left(f_{\mathbb{C}}, \bar{g}_{\mathbb{C}}\right)$ is holomorphic. We define a bilinear function by $B\left(h_{1}, h_{2}\right)=\left\langle h_{1}, \bar{h}_{2}\right\rangle$. Since the composition of holomorphic functions is holomorphic (and a bilinear function is holomorphic), we have that if $h_{1}$ and $h_{2}$ are holomorphic, then $B\left(h_{1}, h_{2}\right)$ is. We thus have that $\left\langle f_{\mathbb{C}}, \overline{\bar{g}_{\mathbb{C}}}\right\rangle$ is holomorphic. Restricted to $\Omega$, this function equals $\langle f, g\rangle$. This shows that $\langle f, g\rangle$ is real-analytic.

The above theorem in particular shows that the squared norm of a real-analytic function is real-analytic, which implies that the squared norm of a holomorphic function is real-analytic. This gives the following corollary.

COROLlARY 9.4. Using the notation and assumptions of Lemma 3.12, we have that $(x, y) \mapsto\|\mathbf{G}(x+i y) u\|^{2}$ and $(x, y) \mapsto 2 x\left\|L_{C}^{1 / 2} \widehat{\mathcal{B}}(x+i y) u\right\|^{2}$ are real-analytic. We quote the following identity theorem for real-analytic functions. This is used in Lemmas 3.12 and 5.4 with $A$ the right half-plane and $U$ some right half-plane.

LEMmA 9.5. Let $A \subset \mathbb{R}^{2}$ be an open connected set, and let $f$ and $g$ be two realanalytic functions in $A$ with values in $E$. If there is a nonempty open subset $U$ of $A$ such that $f(x)=g(x)$ in $U$, then $f(x)=g(x)$ for every $x \in A$. 
Proof. This follows from [12, subsection 9.4.2, p. 208].

Acknowledgments. The authors are grateful to Hans Zwart for lively discussions on Lemma 3.4, to Kalle Mikkola and Amol Sasane for their useful comments, and to Erik Thomas for a discussion on real-analytic functions. We are very grateful to the two anonymous reviewers for their painstaking work in reviewing our manuscript. Their efforts have improved this paper considerably.

\section{REFERENCES}

[1] J. A. Ball, K. M. Miknola, And A. J. Sasane, State-space formulas for the Nehari-Takagi problem for nonexponentially stable infinite-dimensional systems, SIAM J. Control Optim., 44 (2005), pp. 531-563.

[2] R. F. CURTAIN AND A. RAN, Explicit formulas for Hankel norm approximations of infinitedimensional systems, Integral Equations Operator Theory, 13 (1989), pp. 455-469.

[3] R. F. Curtain and H. J. Zwart, The Nehari problem for the Pritchard-Salamon class of infinite-dimensional linear systems: A direct approach, Integral Equations Operator Theory, 18 (1994), pp. 130-153.

[4] R. F. Curtain and H. J. Zwart, An Introduction to Infinite-Dimensional Linear Systems Theory, Springer-Verlag, New York, 1995.

[5] R. F. Curtain And A. IChikawa, The Nehari problem for infinite-dimensional systems of parabolic type, Integral Equations Operator Theory, 26 (1996), pp. 29-45.

[6] R. F. Curtain and J. C. Oostveen, The Nehari problem for nonexponentially stable systems, Integral Equations Operator Theory, 31 (1998), pp. 307-320.

[7] R. F. Curtain and J. C. Oostveen, Necessary and sufficient conditions for strong stability of distributed parameter systems, Systems Control Lett., 37 (1999), pp. 11-18.

[8] R. F. Curtain and A. J. Sasane, Compactness and nuclearity of the Hankel operator and internal stability of infinite-dimensional state linear systems, Internat. J. Control, 74 (2001), pp. $1260-1270$.

[9] R. F. Curtain And A. J. Sasane, Hankel norm approximation for well-posed linear systems, Systems Control Lett., 48 (2003), pp. 407-414.

[10] R. F. Curtain, Regular linear systems and their reciprocals: Applications to Riccati equations, Systems Control Lett., 49 (2003), pp. 81-89.

[11] R. F. CuRTAIn, Riccati equations for stable well-posed linear systems: The generic case, SIAM J. Control Optim., 42 (2003), pp. 1681-1702.

[12] J. Dieudonné, Foundations of Modern Analysis, Academic Press, New York, 1969.

[13] C. Foias And A. TAnnenbaum, On the Nehari problem for a certain class of $L_{\infty}$-functions appearing in control theory, J. Funct. Anal., 74 (1987), pp. 146-159.

[14] K. Glover, R. F. Curtain, and J. R. Partington, Realisation and approximation of linear infinite-dimensional systems with error bounds, SIAM J. Control Optim., 26 (1988), pp. $863-898$.

[15] P. Grabowski, On the spectral-Lyapunov approach to parametric optimization of distributed parameter systems, IMA J. Math. Control Inform., 7 (1991), pp. 317-338.

[16] S. Hansen And G. Weiss, New results on the operator Carleson measure criterion, IMA J. Math. Control Inform., 14 (1997), pp. 3-32.

[17] E. Hille And R. S. Phillips, Functional Analysis and Semi-groups, Amer. Math. Soc. Colloq. Publ., 31, AMS, Providence, RI, 1957.

[18] A. Kheifets, Parametrization of solutions of the Nehari problem and nonorthogonal dynamics, in Operator Theory and Interpolation (Bloomington, IN, 1996), Oper. Theory Adv. Appl. 115, Birkhäuser, Basel, 2000, pp. 213-233.

[19] K. Mikkola, Infinite-Dimensional Linear Systems, Optimal Control, and Riccati Equations, Ph.D. thesis, Helsinki University of Technology, Helsinki, Finland, 2002; also available at http://www.math.hut.fi/reports.

[20] N. K. NIKOL'SkII, Treatise on the Shift Operator, Springer-Verlag, Berlin, 1986.

[21] J. Oostveen, Strongly Stabilizable Distributed Parameter Systems, Frontiers Appl. Math. 20, SIAM, Philadelphia, 2000.

[22] L. B. PAGE, Bounded and compact vectorial Hankel operators, Trans. Amer. Math. Soc., 150 (1970), pp. 529-539.

[23] V. V. Peller, Hankel Operators and Their Applications, Springer Monogr. Math., SpringerVerlag, New York, 2003. 
[24] A. RAN, Hankel norm approximation for infinite-dimensional systems and Wiener-Hopf factorization, in Modelling, Robustness and Sensitivity Reduction in Control Systems, NATO, Adv. Sci. Inst. Ser. F Comput. Systems Sci. 34, R. F. Curtain, ed., Springer-Verlag, Berlin, 1986, pp. 57-69.

[25] M. Rosenblum and J. Rovnyak, Hardy Classes and Operator Theory, Oxford Math. Monogr., The Clarendon Press, Oxford University Press, New York, 1985.

[26] D. Salamon, Realization theory in Hilbert space, Math. Systems Theory, 21 (1989), pp. 147164.

[27] D. Sarason, Generalized interpolation in $H^{\infty}$, Trans. Amer. Math. Soc., 127 (1967), pp. 179203.

[28] A. J. Sasane, Hankel Norm Approximation for Infinite-Dimensional Systems, Lecture Notes in Control and Inform. Sci. 277, Springer-Verlag, Berlin, 2002.

[29] O. J. Staffans, Well-Posed Linear Systems, Cambridge University Press, Cambridge, UK, 2005.

[30] O. J. Staffans, Coprime factorizations and well-posed linear systems, SIAM J. Control Optim., 36 (1998), pp. 1268-1292.

[31] M. Weiss AND G. Weiss, Optimal control of stable weakly regular linear systems, Math. Control Signals Systems, 10 (1997), pp. 287-330.

[32] G. WEIss, Regular linear systems with feedback, Math. Control Signals Systems, 7 (1994), pp. 23-57.

[33] H.J. ZWART, Transfer functions for infinite-dimensional systems, Systems Control Lett., 52 (2004), pp. 247-255. 